\title{
ON SOME FUNDAMENTAL RESULTS ABOUT HIGHER-RANK GRAPHS AND THEIR $C^{*}$-ALGEBRAS
}

\author{
ROBERT HAZLEWOOD, IAIN RAEBURN, AIDAN SIMS \\ AND SAMUEL B.G. WEBSTER
}

\begin{abstract}
Results of Fowler and Sims show that every $k$-graph is completely determined by its $k$-coloured skeleton and collection of commuting squares. Here we give an explicit description of the $k$-graph associated to a given skeleton and collection of squares and show that two $k$-graphs are isomorphic if and only if there is an isomorphism of their skeletons which preserves commuting squares. We use this to prove directly that each $k$-graph $\Lambda$ is isomorphic to the quotient of the path category of its skeleton by the equivalence relation determined by the commuting squares, and show that this extends to a homeomorphism of infinite-path spaces when the $k$-graph is row finite with no sources. We conclude with a short direct proof of the characterisation, originally due to Robertson and Sims, of simplicity of the $C^{*}$-algebra of a row-finite $k$-graph with no sources.
\end{abstract}

\section{INTRODUCTION}

A $k$-graph is a combinatorial object akin to a directed graph, in which each path $\lambda$ has a $k$-dimensional shape $d(\lambda) \in \mathbb{N}^{k}$, called its degree, instead of a 1-dimensional length. $C^{*}$-algebras associated to graphs and $k$-graphs have attracted significant attention recently because they at once encompass a great many interesting examples [2, 6, 11, 17], and are remarkably tractable [3, 4, 5, 7, 12, 13, 21]. Indeed, Spielberg [25] showed how to construct every Kirchberg algebra from combinations of graph $C^{*}$-algebras and 2-graph $C^{*}$-algebras. However, $k$-graphs themselves are, from a combinatorial point of view, substantially more complicated than their 1-dimensional counterparts, and one of the keys to using them effectively is a good visual description.

A crucial feature of $k$-graphs is the factorisation property, which says that, given any path $\lambda$ and any decomposition $d(\lambda)=m+n$, there is a unique factorisation $\lambda=\mu \nu$ such that $d(\mu)=m$ and $d(\nu)=n$. In particular, writing $e_{1}, \ldots, e_{k}$ for the generators of $\mathbb{N}^{k}$, if ef is a path with $d(e)=e_{i}$ and $d(f)=e_{j}$, then $d(e f)=e_{j}+e_{i}$ so there is a unique expression ef $=f^{\prime} e^{\prime}$ where $d\left(f^{\prime}\right)=e_{j}$ and $d\left(e^{\prime}\right)=e_{i}$. This is called a square of $\Lambda$. We can regard the list $\mathcal{C}_{\Lambda}$ of all such squares as data associated with the skeleton of $\Lambda$, which is the $k$-coloured directed graph $E_{\Lambda}$ with the same vertices as $\Lambda$ and with edges $\bigcup_{i=1}^{k} d^{-1}\left(e_{k}\right)$, where edges of different degrees are coloured with different colours.

Theorem 2.2 of [9] characterises exactly which coloured graphs $E$ and collections $\mathcal{C}$ of squares arise from $k$-graphs; and [9, Theorem 2.1] implies that for each such pair $(E, \mathcal{C})$ there is a unique $k$-graph up to isomorphism whose skeleton is $E$ and whose commuting

Date: 8 October 2011.

1991 Mathematics Subject Classification. Primary 05C20; Secondary 46L05.

Key words and phrases. higher-rank graph, $C^{*}$-algebra, Cuntz-Krieger algebra. 
squares are those in $\mathcal{C}$. The latter theorem is an existence result; it does not explicitly describe the $k$-graph $\Lambda_{E, \mathcal{C}}$. It is more or less folklore (and can be dug out of the proof of [9, Theorem 2.1]) that $\Lambda_{E, \mathcal{C}}$ can be described along the lines outlined for $k=2$ in [14, Section 6]: paths in $\Lambda_{E, \mathcal{C}}$ are described as paths in $E$ in which the colours occur in a fixed preferred order. But this is unsatisfactory because it is difficult to recognise a path when it is written as a concatenation of sub-paths, or to decide when one path is a sub-path of another; to do so requires tedious calculations using the collection $\mathcal{C}$ of squares.

In Section 4 we provide a concrete description of the $k$-graph $\Lambda_{E, \mathcal{C}}$. Inspired by the construction of 2-graphs from two-dimensional shift-spaces in [18], we show that the paths in $\Lambda$ can be regarded as coloured-graph morphisms from a collection of model $k$-coloured graphs into $E$. An advantage of this construction is that under this presentation, each path explicitly encodes all of its subpaths. In Section 5 we use this to provide an explicit proof that $\Lambda$ is the quotient of the path category $E_{\Lambda}^{*}$ of $E_{\Lambda}$ by the equivalence relation $\sim$ determined by $\mathcal{C}$. We then show that the topology on the infinitepath space of $\Lambda$ coincides with the quotient topology on $E^{\infty} / \sim$. We also present an example showing that the corresponding statement is false for boundary paths in nonrow-finite $k$-graphs. Our final section gives a direct and elementary proof that if $\Lambda$ is a row-finite $k$-graph with no sources, then $C^{*}(\Lambda)$ is simple if and only $\Lambda$ is both aperiodic and cofinal (see Section 6 for details). This result first appeared in [21], but the proof there was indirect, proceeding via reference to the results of [14], which were proved using groupoid technology. Since aperiodicity and cofinality have been characterised in a number of different ways in the literature, we use the presentations which are best suited to the description of $\Lambda_{E, \mathcal{C}}$ from Section 4: specifically, the description of aperiodicity introduced in [21], and the cofinality condition of [15]. The key graph-theoretic component, Lemma 6.2, of our proof has already found applications elsewhere: it was precisely the statement needed to establish the Cuntz-Krieger uniqueness theorem [1, Theorem 4.7] for the Kumjian-Pask algebras introduced there.

\section{BACKGROUND}

A directed graph $E=\left(E^{0}, E^{1}, r, s\right)$ consists of countable sets $E^{0}, E^{1}$ and functions $r, s: E^{1} \rightarrow E^{0}$. Since all the graphs in this paper are directed, we will drop the adjective. We call elements of $E^{0}$ vertices, and elements of $E^{1}$ edges. For an edge $e \in E^{1}$, we call $s(e)$ the source of $e$ and $r(e)$ the range of $e$. A path of length $n$ is a sequence $\mu=\mu_{1} \mu_{2} \ldots \mu_{n}$ of edges such that $s\left(\mu_{i}\right)=r\left(\mu_{i+1}\right)$ for $1 \leq i \leq n-1$. We denote by $E^{n}$ the set of all paths of length $n$, and define $E^{*}:=\bigcup_{n \in \mathbb{N}} E^{n}$. We extend $r$ and $s$ to $E^{*}$ by setting $r(\mu)=r\left(\mu_{1}\right)$ and $s(\mu)=s\left(\mu_{n}\right)$. By an infinite path in $E$, we mean a sequence $x=\nu_{1} \nu_{2} \ldots$ where $r\left(\nu_{i+1}\right)=s\left(\nu_{i}\right)$ for all $i$, and we write $r(x)=r\left(\nu_{1}\right)$. We write $E^{\infty}$ for the set of all infinite paths, and call $W_{E}:=E^{*} \cup E^{\infty}$ the path space of E.

For $k \in \mathbb{N}$, a $k$-graph is a pair $(\Lambda, d)$ where $\Lambda$ is a countable category and $d$ is a functor from $\Lambda$ to $\mathbb{N}^{k}$ which satisfies the factorisation property: for every $\lambda \in \operatorname{Mor}(\Lambda)$ and $m, n \in \mathbb{N}^{k}$ with $d(\lambda)=m+n$, there are unique elements $\mu, \nu \in \operatorname{Mor}(\Lambda)$ such that $\lambda=\mu \nu, d(\mu)=m$ and $d(\nu)=n$ (see [14, Definition 1.1]). Elements $\lambda \in \operatorname{Mor}(\Lambda)$ are 
called paths, and by convention we write $\lambda \in \Lambda$ to mean $\lambda \in \operatorname{Mor}(\Lambda)$. The functor $d$ is called the degree map.

For $m \in \mathbb{N}^{k}$ and $v \in \operatorname{Obj}(\Lambda)$, we define $\Lambda^{m}:=\{\lambda \in \Lambda: d(\lambda)=m\}$ and $v \Lambda^{m}:=\{\lambda \in$ $\left.\Lambda^{m}: r(\lambda)=v\right\}$. More generally, given $\lambda \in \Lambda$ and $F, G \subseteq \Lambda$, we define $\lambda G=\{\lambda \nu: \nu \in$ $G, r(\nu)=s(\lambda)\}$ and $F \lambda=\{\mu \lambda: \mu \in F, s(\mu)=r(\lambda)\}$; and then $F \lambda G=\bigcup_{\mu \in F} \mu \lambda G=$ $\bigcup_{\nu \in G} F \lambda \nu$.

A morphism between $k$-graphs $\left(\Lambda_{1}, d_{1}\right)$ and $\left(\Lambda_{2}, d_{2}\right)$ is a functor $f: \Lambda_{1} \rightarrow \Lambda_{2}$ which respects the degree maps. The factorisation property implies that $v \mapsto \mathrm{id}_{v}$ is a bijection between $\operatorname{Obj}(\Lambda)$ and $\Lambda^{0}$, allowing us to identify $\operatorname{Obj}(\Lambda)$ with $\Lambda^{0}$. In particular, we will henceforth regard $r$ and $s$ as maps from $\Lambda$ to $\Lambda^{0}$.

\section{COLOURED GRAPHS AND COLOURED-GRAPH MORPHISMS}

Consider the free semigroup $\mathbb{F}_{k}$ on $k$-generators $\left\{c_{1}, \ldots c_{k}\right\}$. A $k$-coloured graph is a graph $E$ together with a map $c: E^{1} \rightarrow\left\{c_{1}, \ldots, c_{k}\right\}$, which we extend to a functor $c: E^{*} \rightarrow \mathbb{F}_{k}^{+}$. We write $q$ for the canonical quotient map $q: \mathbb{F}_{k}^{+} \rightarrow \mathbb{N}^{k}$ determined by $q\left(c_{i}\right)=e_{i}$ for all $i$. So each path $x \in E^{*}$ has both a colouring $c(x) \in \mathbb{F}_{k}^{+}$and a shape $q(c(x)) \in \mathbb{N}^{k}$. If there are multiple $k$-coloured graphs around, we write $c_{E}$ for the colour map associated to the graph $E$. In this paper, we will draw edges of colour $c_{1}$ in blue and solid, edges of colour $c_{2}$ in red and dashed, and edges of colour $c_{3}$ in green and dotted.

A graph morphism $\psi$ from a graph $E$ to a graph $F$ consists of functions $\psi^{0}: E^{0} \rightarrow F^{0}$ and $\psi^{1}: E^{1} \rightarrow F^{1}$ such that $r_{F}\left(\psi^{1}(e)\right)=\psi^{0}\left(r_{E}(e)\right)$ and $s_{F}\left(\psi^{1}(e)\right)=\psi^{0}\left(s_{E}(e)\right)$ for all $e \in E^{1}$. Given graph morphisms $\psi: E \rightarrow F$ and $\phi: F \rightarrow G$, we write $\phi \circ \psi$ for the graph morphism from $E$ to $G$ given by $(\phi \circ \psi)^{i}=\phi^{i} \circ \psi^{i}$ for $i=0,1$. A coloured-graph morphism is a graph morphism $\psi$ such that $c_{E}(e)=c_{F}(\psi(e))$ for every $e \in E^{1}$.

The following example describes the model $k$-coloured graphs which will underly the construction used in our main theorem in Section 4. In the example, $n+v_{i}$ is a formal symbol intended to suggest an edge of colour $c_{i}$ pointing from the integer-grid point $n+e_{i}$ to the integer-grid point $n$.

Example 3.1. For $m \in(\mathbb{N} \cup\{\infty\})^{k}$, we define a coloured graph $E_{k, m}$ by

$$
\begin{gathered}
E_{k, m}^{0}=\left\{n \in \mathbb{N}^{k}: 0 \leq n \leq m\right\}, \quad E_{k, m}^{1}=\left\{n+v_{i}: n, n+e_{i} \in E_{k, m}^{0}\right\}, \\
r\left(n+v_{i}\right)=n, \quad s\left(n+v_{i}\right)=n+e_{i} \quad \text { and } \quad c\left(n+v_{i}\right)=c_{i} .
\end{gathered}
$$

For $n+v_{i} \in E^{1}$ and $m \in \mathbb{N}^{k}$, we define $\left(n+v_{i}\right)+m:=(n+m)+v_{i}$. For $x \in E^{1}$ with $c(x)=c_{j}$, it is unambiguous and often useful to write $v_{c(x)}:=v_{j}$. Given a colouredgraph morphism $\lambda: E_{k, m} \rightarrow E$ we say $\lambda$ has degree $m$ and write $d(\lambda)=m$, and define $r(\lambda):=\lambda(0)$ and $s(\lambda):=\lambda(m)$.

Given a $k$-coloured graph $E$ and distinct $i, j \in\{1, \ldots, k\}$, an $\{i, j\}$-square (or just a square) in $E$ is a coloured-graph morphism $\phi: E_{k, e_{i}+e_{j}} \rightarrow E$. If $\lambda: E_{k, m} \rightarrow E$ is a coloured-graph morphism and $\phi$ is a square in $E$, then $\phi$ occurs in $\lambda$ if there exists $n \in \mathbb{N}^{k}$ such that $\phi(x)=\lambda(x+n)$ for all $x \in E_{k, e_{i}+e_{j}}$.

Let $E$ be a $k$-coloured graph. A complete collection of squares is a collection $\mathcal{C}$ of squares in $E$ such that for each $x \in E^{*}$ with $c(x)=c_{i} c_{j}$ and $i \neq j$, there exists a unique $\phi \in \mathcal{C}$ such that $x=\phi\left(v_{i}\right) \phi\left(e_{i}+v_{j}\right)$. We write $\phi\left(v_{i}\right) \phi\left(e_{i}+v_{j}\right) \sim_{\mathcal{C}} \phi\left(v_{j}\right) \phi\left(e_{j}+v_{i}\right)$, so 
for each $c_{i} c_{j}$-coloured path $x \in E^{*}$, there is a unique $c_{j} c_{i}$-coloured path $y$ such that $x \sim_{\mathcal{C}} y$. If $\mathcal{C}$ is clear from context, we just write $x \sim y$. A coloured-graph morphism $\lambda: E_{k, m} \rightarrow E$ is $\mathcal{C}$-compatible if every square occurring in $\lambda$ belongs to $\mathcal{C}$.

For $p, q \in \mathbb{N}^{k}$ with $p \leq q$, define $E_{k,[p, q]}$ to be the subgraph of $E_{k, q}$ such that

$$
\begin{aligned}
& E_{k,[p, q]}^{0}=\left\{n \in \mathbb{N}^{k}: p \leq n \leq q\right\}, \\
& E_{k,[p, q]}^{1}=\left\{x \in E_{k, q}^{1}: s(x), r(x) \in E_{k,[p, q]}^{0}\right\} .
\end{aligned}
$$

Given a coloured-graph morphism $\lambda: E_{k, m} \rightarrow E$ and $p, q \in \mathbb{N}^{k}$ such that $p \leq q \leq m$, define $\left.\lambda\right|_{E_{k,[p, q]}} ^{*}: E_{k, q-p} \rightarrow E$ by

$$
\left.\lambda\right|_{E_{k,[p, q]}} ^{*}(a)=\lambda(p+a) .
$$

The star is to remind us that this non-standard restriction involves a translation.

We say a complete collection of squares $\mathcal{C}$ in a $k$-coloured graph $E$ is associative if for every path $f g h$ in $E$ such that $f, g, h$ are edges of distinct colour, the edges $f_{1}, f_{2}, g_{1}, g_{2}, h_{1}, h_{2}$ and $f^{1}, f^{2}, g^{1}, g^{2}, h^{1}, h^{2}$ determined by

$$
\begin{aligned}
& f g \sim g^{1} f^{1}, f^{1} h \sim h^{1} f^{2}, \text { and } g^{1} h^{1} \sim h^{2} g^{2} \\
& g h \sim h_{1} g_{1}, f h_{1} \sim h_{2} f_{1}, \text { and } f_{1} g_{1} \sim g_{2} f_{2}
\end{aligned}
$$

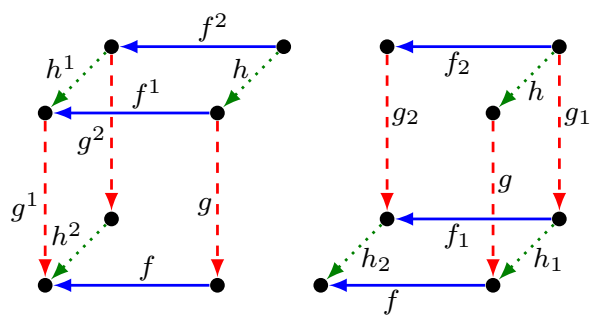

satisfy $f^{2}=f_{2}, g^{2}=g_{2}$ and $h^{2}=h_{2}$.

Let $E$ be a $k$-coloured graph, and $m \in \mathbb{N}^{k} \backslash\{0\}$. Fix $x \in E^{*}$ and a coloured-graph morphism $\lambda: E_{k, m} \rightarrow E$. We say $x$ traverses $\lambda$ if the shape $q(c(x))$ of $x$ is equal to $d(\lambda)$ and $\lambda\left(q\left(c\left(x_{1} \ldots x_{l-1}\right)\right)+v_{c\left(x_{l}\right)}\right)=x_{l}$ for all $0<l \leq|m|$. If $m=0$, then $x \in E^{0}$ and $\operatorname{dom}(\lambda)=\{0\}$, and we say $x$ traverses $\lambda$ if $x=\lambda(0)$. Observe that for any coloured-graph morphism $\lambda$, and any decomposition $d(\lambda)=e_{i_{1}}+e_{i_{2}}+\cdots+e_{i_{|\lambda|}}$ there is a corresponding path $x:=\lambda\left(0+v_{i_{1}}\right) \lambda\left(e_{i_{1}}+v_{i_{2}}\right) \ldots \lambda\left(\left(d(\lambda)-e_{i_{|\lambda|}}\right)+v_{i_{|\lambda|}}\right)$ which traverses $\lambda$; in particular, for every finite coloured-graph morphism $\lambda$ there is a path which traverses $\lambda$.

We can also make sense of infinite coloured paths which traverse infinite colouredgraph morphisms. If $x \in E^{\infty}$ and $\lambda: E_{k, p} \rightarrow E$ is a coloured-graph morphism of non-finite degree (so $\left.p \in(\mathbb{N} \cup\{\infty\})^{k} \backslash \mathbb{N}^{k}\right)$, then we say that $x$ traverses $\lambda$ if $x_{1} \ldots x_{n}$ traverses $\left.\lambda\right|_{E_{k, d\left(x_{1} \ldots x_{n}\right)}}$ for every $n \in \mathbb{N}$.

Remark 3.2. Let $E$ be a $k$-coloured graph and let $\lambda: E_{k, m} \rightarrow E$ be a coloured-graph morphism where $m \in \mathbb{N}^{k}$. Fix $p \leq m$. If $x \in E^{*}$ traverses $\left.\lambda\right|_{[0, p]}$ and $y \in E^{*}$ traverses of $\left.\lambda\right|_{[p, m]} ^{*}$, then $d(\lambda)=m=p+(m-p)=q(c(x))+q(c(y))$, and for $l \leq|x y|=|x|+|y|$, we have

$$
\begin{aligned}
\lambda\left(d\left((x y)_{1} \ldots(x y)_{l-1}\right)+v_{c\left((x y)_{l}\right)}\right) & = \begin{cases}\left.\lambda\right|_{[0, p]}\left(q\left(c\left(x_{1} \ldots x_{l-1}\right)\right)+v_{\left.c(x)_{l}\right)}\right) & \text { if } l \leq|x| \\
\left.\lambda\right|_{[p, m]} ^{*}\left(q\left(c\left(y_{1} \ldots y_{l-p-1}\right)\right)+v_{c\left(y_{l-p}\right)}\right) & \text { otherwise }\end{cases} \\
& = \begin{cases}x_{l} & \text { if } l \leq|x| \\
y_{l-p} & \text { otherwise, }\end{cases}
\end{aligned}
$$


so $x y$ traverses $\lambda$.

\section{From $k$-COLOURED GRAPHS TO $k$-GRAPHS.}

In this section we present an explicit description of the unique $k$-graph associated to a $k$-coloured graph $E$ and complete collection $\mathcal{C}$ of squares in $E$ which is associative (see Theorem 4.4).

We begin by showing how a $k$-graph defines a skeleton and a collection of squares. If $\Lambda$ is a $k$-graph, $\lambda \in \Lambda$, and $m \leq n \leq d(\lambda)$, then we write $\lambda(m, n)$ for the unique element of $\Lambda^{m-n}$ such that $\lambda=\lambda^{\prime} \lambda(m, n) \lambda^{\prime \prime}$ with $d\left(\lambda^{\prime}\right)=m$ and $d\left(\lambda^{\prime \prime}\right)=d(\lambda)-n$. We write $\lambda(n)$ for $s(\lambda(0, n)) \in \Lambda^{0}$.

Definition 4.1. Let $\Lambda$ be a $k$-graph. We define a coloured graph $E_{\Lambda}$ and a collection $\mathcal{C}_{\Lambda}$ of squares associated to $\Lambda$ as follows. Let $E_{\Lambda}$ be the $k$-coloured graph with $E_{\Lambda}^{0}=\{\bar{v}$ : $\left.v \in \Lambda^{0}\right\}, E_{\Lambda}^{1}=\bigcup_{i=1}^{k}\left\{\bar{f}: f \in \Lambda^{e_{i}}\right\}$, and $c(\bar{f})=c_{i} \Longleftrightarrow d(f)=e_{i}$. Define $\pi: E_{\Lambda}^{0} \rightarrow \Lambda$ by $\pi(\bar{v})=v$ and $\pi: E_{\Lambda}^{1} \rightarrow \Lambda$ by $\pi(\bar{f})=f$, and extend this to a map $\pi: E_{\Lambda}^{*} \rightarrow \Lambda$ by $\pi\left(\overline{f_{1}} \ldots \overline{f_{n}}\right)=f_{1} \cdots f_{n}$. For distinct $i, j \leq k$ and $\lambda \in \Lambda^{e_{i}+e_{j}}$ define a coloured-graph morphism $\phi_{\lambda}: E_{k, e_{i}+e_{j}} \rightarrow E_{\Lambda}$ by

$$
\phi_{\lambda}^{0}(n)=\overline{\lambda(n)} \quad \text { and } \quad \phi_{\lambda}^{1}\left(n+v_{i}\right):=\overline{\lambda\left(n, n+e_{i}\right)} .
$$

Let $\mathcal{C}_{\Lambda}:=\bigcup_{i<j \leq k}\left\{\phi_{\lambda}: \lambda \in \Lambda^{e_{i}+e_{j}}\right\}$. We call $E_{\Lambda}$ the skeleton of $\Lambda$.

Lemma 4.2. Let $\Lambda$ be a k-graph. Fix distinct $i, j \leq k$ and $\lambda \in \Lambda^{e_{i}+e_{j}}$. Then $\phi_{\lambda}$ is the unique coloured-graph morphism from $E_{k, e_{i}+e_{j}} \rightarrow E_{\Lambda}$ such that

$$
\pi\left(\phi_{\lambda}\left(0+v_{i}\right) \phi_{\lambda}\left(e_{i}+v_{j}\right)\right)=\lambda=\pi\left(\phi_{\lambda}\left(0+v_{j}\right) \phi_{\lambda}\left(e_{j}+v_{i}\right)\right)
$$

Moreover $\mathcal{C}_{\Lambda}$ is a complete collection of squares in $E_{\Lambda}$ which is associative.

Proof. Fix distinct $i, j \leq k$, and $\lambda \in \Lambda^{e_{i}+e_{j}}$. Then

$$
\pi\left(\phi_{\lambda}\left(0+v_{i}\right) \phi_{\lambda}\left(e_{i}+v_{j}\right)\right)=\pi\left(\overline{\lambda\left(0, e_{i}\right)} \overline{\lambda\left(e_{i}, e_{i}+e_{j}\right)}\right)=\lambda\left(0, e_{i}\right) \lambda\left(e_{i}, e_{i}+e_{j}\right)=\lambda .
$$

The symmetric calculation shows that $\pi\left(\phi_{\lambda}\left(0+v_{j}\right) \phi_{\lambda}\left(e_{j}+v_{i}\right)\right)=\lambda$ also. Hence $\phi_{\lambda}$ satisfies (4.2). To see that it is the unique such coloured-graph morphism, suppose that $f \in c^{-1}(i)$ and $g \in c^{-1}(j)$ and $\pi(f g)=\lambda$. Then the factorisation property forces $\pi(f)=\lambda\left(0, e_{i}\right)$ and $\pi(g)=\lambda\left(e_{i}, e_{i}+e_{j}\right)$. Since $\pi$ is injective on $E_{\Lambda}^{1}$, it follows that $f=\overline{\lambda\left(0, e_{i}\right)}=\phi_{\lambda}\left(0+v_{i}\right)$ and $g=\overline{\lambda\left(e_{i}, e_{i}+e_{j}\right)}=\phi_{\lambda}\left(e_{i}+v_{j}\right)$. A symmetric argument applies with $i$ and $j$ interchanged, and this proves the first statement of the lemma.

To see that the collection $\mathcal{C}_{\Lambda}$ is complete, fix $f, g \in E_{\Lambda}^{1}$ with $s(f)=r(g)$ and $c(f) \neq$ $c(g)$, say $c(f)=c_{i}$ and $c(g)=c_{j}$. Then $\pi(f) \in \Lambda^{e_{i}}$ and $\pi(g) \in \Lambda^{e_{j}}$, so $\pi(f g) \in \Lambda^{e_{i}+e_{j}}$, and the factorisation property ensures that $f g$ traverses $\phi_{\pi(f g)}$. Moreover, if $\lambda \in \Lambda^{e_{i}+e_{j}}$ is another path such that $f g$ traverses $\phi_{\lambda}$, then

$$
\lambda=\lambda\left(0, e_{i}\right) \lambda\left(e_{i}, e_{i}+e_{j}\right)=\pi\left(\phi_{\lambda}\left(0+v_{i}\right) \phi_{\lambda}\left(e_{i}+v_{j}\right)\right)=\pi(f g),
$$

so $\phi_{\pi(f g)}$ is the unique element of $\mathcal{C}_{\Lambda}$ such that $f g$ traverses $\phi_{\pi(f g)}$. For the associativity condition, suppose we have $f, g, h, f^{i}, g^{i}, h^{i}$, and $f_{i}, g_{i}, h_{i}$ as in (3.2). By associativity of composition in $\Lambda$, we have

$$
\pi\left(h_{2} g_{2} f_{2}\right)=\pi(f g h)=\pi\left(h^{2} g^{2} f^{2}\right)
$$


so the factorisation property in $\Lambda$ forces $\pi\left(h_{2}\right)=\pi\left(h^{2}\right), \pi\left(g_{2}\right)=\pi\left(g^{2}\right)$ and $\pi\left(f_{2}\right)=\pi\left(f^{2}\right)$. Since $\pi$ is injective on $E_{\Lambda}^{1}$, it follows that $h_{2}=h^{2}, g_{2}=g^{2}$ and $f_{2}=f^{2}$ as required.

Notation 4.3. Let $E$ be a $k$-coloured graph, and let $\mathcal{C}$ be a complete collection of squares in $E$ which is associative. For each $m \in \mathbb{N}^{k}$, we write $\Lambda_{(E, \mathcal{C})}^{m}$ for the set of all $\mathcal{C}$-compatible coloured-graph morphisms $E_{k, m} \rightarrow E$. Let $\Lambda_{(E, \mathcal{C})}:=\bigcup_{m \in \mathbb{N}^{k}} \Lambda_{(E, \mathcal{C})}^{m}$. Let $d: \Lambda_{(E, \mathcal{C})} \rightarrow \mathbb{N}^{k}$ and $r, s: \Lambda_{(E, \mathcal{C})} \rightarrow \Lambda_{E}^{0}$ be as defined in Example 3.1. For $v \in E^{0}$ we define $\lambda_{v}: E_{k, 0} \rightarrow E$ by $\lambda_{v}(0)=v$, and for $1 \leq i \leq k$ and $f \in E^{1}$ with $c(f)=c_{i}$ we define $\lambda_{f}: E_{k, e_{i}} \rightarrow E$ by $\lambda_{f}(0)=r(f), \lambda_{f}\left(e_{i}\right)=s(f)$ and $\lambda_{f}\left(0+v_{i}\right)=f$.

Our first main theorem shows that the notation above describes a $k$-graph whose skeleton is isomorphic to $E$ under an isomorphism which carries the commuting squares of $\Lambda$ to the elements of $\mathcal{C}$.

Theorem 4.4. Fix a $k$-coloured graph $E$ and a complete collection of squares $\mathcal{C}$ in $E$ which is associative. If $\mu: E_{k, m} \rightarrow E$ and $\nu: E_{k, n} \rightarrow E$ are $\mathcal{C}$-compatible coloured-graph morphisms such that $s(\mu)=r(\nu)$, then there exists a unique $\mathcal{C}$-compatible coloured-graph morphism $\mu \nu: E_{k, m+n} \rightarrow E$ such that $\left.(\mu \nu)\right|_{E_{k, m}}=\mu$ and $\left.(\mu \nu)\right|_{E_{k,[m, m+n]}} ^{*}=\nu$. Under this composition map, the set $\Lambda=\Lambda_{(E, \mathcal{C})}$ of Notation 4.3. endowed with the structure maps defined there, is a k-graph. There is an isomorphism $\rho: E \rightarrow E_{\Lambda}$ such that $\rho^{0}(v)=\overline{\lambda_{v}}$ for all $v \in E^{0}$ and $\rho^{1}(f)=\overline{\lambda_{f}}$ for all $f \in E^{1}$; and this $\rho$ satisfies $\rho \circ \phi \in \mathcal{C}_{\Lambda}$ for all $\phi \in \mathcal{C}$.

Our second main theorem says that the $k$-graph $\Lambda_{(E, \mathcal{C})}$ is uniquely determined, up to isomorphism, by the isomorphism class of $(E, \mathcal{C})$.

Theorem 4.5. Fix a $k$-graph $\Gamma$, a $k$-coloured graph $E$ and a complete collection $\mathcal{C}$ of squares in $E$ which is associative. Suppose that $\psi: E_{\Gamma} \rightarrow E$ is a coloured-graph isomorphism such that $\psi \circ \phi \in \mathcal{C}$ for all $\phi \in \mathcal{C}_{\Gamma}$. Then for each $\gamma \in \Gamma$ there is a $\mathcal{C}$-compatible coloured-graph morphism $\theta_{\gamma}: E_{k, d(\gamma)} \rightarrow E$ such that

$$
\begin{aligned}
\theta_{\gamma}^{0}(m) & =\psi^{0}(\overline{\gamma(m)}) \quad \text { for } m \in E_{k, d(\gamma)}^{0}, \text { and } \\
\theta_{\gamma}^{1}\left(m+v_{i}\right) & =\psi^{1}\left(\overline{\gamma\left(m, m+e_{i}\right)}\right) \quad \text { for } m+v_{i} \in E_{k, d(\gamma)}^{1} .
\end{aligned}
$$

Moreover, the map $\theta: \gamma \mapsto \theta_{\gamma}$ is an isomorphism $\Gamma \cong \Lambda_{(E, \mathcal{C})}$.

The key technical result which we need to prove Theorems 4.4 and 4.5 says that every path in the coloured graph $E$ determines a unique element of $\Lambda$. We first use the associativity condition to prove this in the special case of a tri-coloured path of length three, and then deal with arbitrary paths using an inductive argument.

Lemma 4.6. Let $E$ be a $k$-coloured graph and let $\mathcal{C}$ be a complete collection of squares in $E$ which is associative. If $f, g, h \in E^{1}$ are of distinct colour and $f g h$ is a path in $E$, then there is a unique $\mathcal{C}$-compatible coloured-graph morphism $\lambda: E_{k, d(f g h)} \rightarrow E$ such that $f g h$ traverses $\lambda$.

Proof. The completeness of $\mathcal{C}$ implies that there exist paths $f^{i}, g^{i}, h^{i}$ and $f_{i}, g_{i}, h_{i}$ satisfying the equations (3.2). Let $\lambda$ be the coloured-graph morphism such that each of $f g h$, $f h_{1} g_{1}, h_{2} f_{1} g_{1}, h_{2} g_{2} f_{2}, g^{1} f^{1} h$, and $g^{1} h^{1} f^{2}$ traverses $\lambda$. Associativity of $\mathcal{C}$ ensures that $\lambda$ is $\mathcal{C}$-compatible. Since the values of the $f^{i}, g^{i}, h^{i}$ and $f_{i}, g_{i}, h_{i}$ are determined by $f, g, h$ and $\mathcal{C}$, if $f g h$ traverses $\mu$ also, then $\mu=\lambda$. 
Proposition 4.7. Let $E$ be a $k$-coloured graph and let $\mathcal{C}$ be a complete collection of squares in $E$ which is associative. For every $x \in E^{*}$ there is a unique $\mathcal{C}$-compatible coloured-graph morphism $\lambda_{x}: E_{k, d(x)} \rightarrow E$ such that $x$ traverses $\lambda_{x}$.

Remark 4.8. The notation of Proposition 4.7 is consistent with that of Notation 4.3 since $\lambda_{v}$ and $\lambda_{f}$ (see Notation 4.3) are the unique morphisms such that $v$ traverses $\lambda_{v}$ and $f$ traverses $\lambda_{f}$.

Proof of Proposition 4.7. We prove this by induction on $|x|$. If $|x|=0$, then the result is trivial.

Now suppose as an inductive hypothesis that for every $y \in E^{*}$ with $|y| \leq n$, the path $y$ traverses a unique coloured-graph morphism $\lambda_{y}: E_{k, d(y)} \rightarrow E$. Fix a path $x \in E^{*}$ with $|x|=n+1$, and express $x=y f$ where $f \in E^{1}$, with $c(f)=c_{i}$, say.

Let $m:=q(c(y))$. By the inductive hypothesis, $y$ traverses a unique $\mathcal{C}$-compatible coloured-graph morphism $\lambda_{y}$. We complete the proof by consideration of three cases: $\left|\left\{j \neq i: m_{j}>0\right\}\right|=0,\left|\left\{j \neq i: m_{j}>0\right\}\right|=1$, and then $\left|\left\{j \neq i: m_{j}>0\right\}\right| \geq 2$.

Suppose first that $\left|\left\{j \neq i: m_{j}>0\right\}\right|=0$. Then $E_{k, d(x)}=E_{k, m} \cup E_{k,\left[m, m+e_{i}\right]}$, so the formulae

$$
\left.\lambda_{x}\right|_{E_{k, m}}=\lambda_{y}, \quad \lambda_{x}\left(m+v_{i}\right)=f \quad \text { and } \quad \lambda_{x}\left(m+e_{i}\right)=s(f)
$$

completely specify a coloured-graph morphism $\lambda_{x}$ such that $x$ traverses $\lambda_{x}$. Furthermore, $\lambda_{x}$ is the unique such coloured-graph morphism: if $x$ also traverses $\mu$, then $\mu$ satisfies the formulae (4.5). This completes the proof when $\left|\left\{j \neq i: m_{j}>0\right\}\right|=0$.

Suppose for the rest of the proof that $\left|\left\{j \neq i: m_{j}>0\right\}\right| \geq 1$ (we will consider separately later the cases $\left|\left\{j \neq i: m_{j}>0\right\}\right|=1$ and $\left.\left|\left\{j \neq i: m_{j}>0\right\}\right| \geq 2\right)$. Then

$$
E_{k, m+e_{i}}=E_{k, m} \cup\left(\bigcup_{j \neq i, m_{j}>0} E_{k, m+e_{i}-e_{j}}\right) \cup\left(\bigcup_{j \neq i, m_{j}>0} E_{k,\left[m-e_{j}, m+e_{i}\right]}\right) .
$$

For each $j \neq i$ such that $m_{j}>0$, fix, for the remainder of the proof, a path $z^{j}$ which traverses $\left.\lambda_{y}\right|_{E_{k, m-e_{j}}}$.

Claim 1. Suppose that $j \neq i$ satisfies $m_{j}>0$. Let $\phi^{j}$ be the unique square in $\mathcal{C}$ traversed by $\lambda_{y}\left(\left(m-e_{j}\right)+v_{j}\right) f$. Let $g^{j}=\phi^{j}\left(0+v_{i}\right)$ and $h^{j}=\phi^{j}\left(e_{i}+v_{j}\right)$, so $g^{j} h^{j} \sim$ $\lambda_{y}\left(\left(m-e_{j}\right)+v_{j}\right) f$. Then there is a unique coloured-graph morphism $\lambda^{j}: E_{k, m-e_{j}+e_{i}} \rightarrow E$ such that $\left.\lambda^{j}\right|_{E_{k, m-e_{j}}}=\left.\lambda_{y}\right|_{E_{k, m-e_{j}}}$ and $\lambda^{j}\left(\left(m-e_{j}\right)+v_{i}\right)=g^{j}$.

To prove Claim 1, observe that $\left|z^{j} g^{j}\right|=n$, so the inductive hypothesis implies that $z^{j} g^{j}$ traverses a unique $\mathcal{C}$-compatible coloured-graph morphism $\lambda^{j}$. Since $z^{j}$ traverses both $\left.\lambda^{j}\right|_{E_{k, m-e_{j}}}$ and $\left.\lambda_{y}\right|_{E_{k, m-e_{j}}}$, the inductive hypothesis implies that the two are equal. This proves Claim 1,

Suppose now that $\left|\left\{j \neq i: m_{j}>0\right\}\right|=1$; let $j$ be the unique element of this set. Then Claim 1 and (4.6) imply that there is a well-defined function $\lambda_{x}: E_{k, m+e_{i}} \rightarrow E$ such that

$$
\left.\lambda_{x}\right|_{E_{k, m}}=\lambda_{y},\left.\quad \lambda_{x}\right|_{E_{k, m+e_{i}-e_{j}}}=\lambda^{j} \quad \text { and }\left.\quad \lambda_{x}\right|_{E_{k,\left[m-e_{j}, m+e_{i}\right]}} ^{*}=\phi^{j} .
$$

This $\lambda_{x}$ is $\mathcal{C}$-compatible by construction, and $x$ traverses $\lambda_{x}$. For uniqueness, fix a $\mathcal{C}$-compatible coloured-graph morphism $\mu$ traversed by $x$. Then $z^{j} g^{j} h^{j}$ traverses $\mu$. 


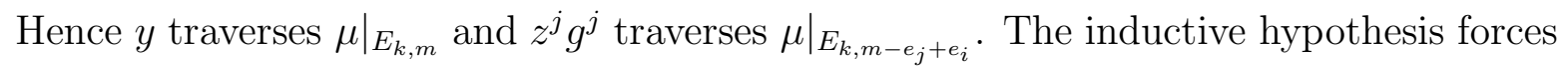
$\left.\mu\right|_{E_{k, m}}=\lambda_{y}$ and $\left.\mu\right|_{E_{k, m-e_{j}+e_{i}}}=\lambda^{j}$. That $\mu$ is $\mathcal{C}$-compatible implies that $\left.\mu\right|_{E_{k,\left[m-e_{j}, m+e_{i}\right]}^{*}} ^{*}=$ $\phi^{j}$. So $\mu=\lambda_{x}$. This proves the lemma when there is a unique $j \neq i$ such that $m_{j} \neq 0$ as claimed.

We now consider the last remaining case: suppose that there are at least two distinct $j, l \neq i$ such that $m_{j}, m_{l}>0$.

Claim 2. For distinct $j, l \neq i$ with $m_{j}, m_{l} \neq 0$, we have $\left.\lambda^{j}\right|_{E_{k, m+e_{i}-e_{j}-e_{l}}}=\left.\lambda^{l}\right|_{E_{k, m+e_{i}-e_{j}-e_{l}}}$.

To establish Claim 2, observe that since $i, j, l$ are all different, Lemma 4.6 implies that $\lambda_{y}\left(\left(m-e_{j}-e_{l}\right)+v_{l}\right) \lambda_{y}\left(\left(m-e_{j}\right)+v_{j}\right) f$ traverses a unique $\mathcal{C}$-compatible graph morphism $\psi^{j, l}$. We show that

$$
\left.\lambda^{j}\right|_{E_{k,\left[m-e_{l}-e_{j}, m+e_{i}-e_{j}\right]}^{*}} ^{*}=\left.\psi^{j, l}\right|_{E_{k, e_{i}+e_{l}}} \text { and }\left.\quad \lambda^{l}\right|_{E_{k,\left[m-e_{l}-e_{j}, m+e_{i}-e_{l}\right]}^{*}} ^{*}=\left.\psi^{j, l}\right|_{E_{k, e_{i}+e_{j}}} .
$$

By symmetry, it suffices to establish that $\left.\lambda^{j}\right|_{E_{k,\left[m-e_{l}-e_{j}, m+e_{i}-e_{j}\right]}^{*}} ^{*}=\left.\psi^{j, l}\right|_{E_{k, e_{i}+e_{l}}}$. Since $\lambda_{y}\left(\left(m-e_{j}\right)+v_{j}\right) f \sim g^{j} h^{j}$, and since $\psi^{j, l}$ is a $\mathcal{C}$-compatible coloured-graph morphism, $\lambda_{y}\left(\left(m-e_{j}-e_{l}\right)+v_{l}\right) g^{j} h^{j}=\lambda^{j}\left(\left(m-e_{j}-e_{l}\right)+v_{l}\right) \lambda^{j}\left(\left(m-e_{j}\right)+v_{i}\right) h^{j} \quad$ traverses $\psi^{j, l}$. Since $\mathcal{C}$ is a complete collection of squares, $\left.\lambda^{j}\right|_{E_{k,\left[m-e_{l}-e_{j}, m+e_{i}-e_{j}\right]}^{*}} ^{*}=\left.\psi^{j, l}\right|_{E_{k, e_{i}+e_{l}}}$. This proves (4.8).

To complete the proof of Claim 2, note that

$$
\left.\lambda^{j}\right|_{E_{k, m-e_{j}-e_{l}}}=\left.\lambda_{y}\right|_{E_{k, m-e_{j}-e_{l}}}=\left.\lambda^{l}\right|_{E_{k, m-e_{j}-e_{l}}} .
$$

Suppose that $z$ traverses this morphism. Equation (4.8) implies that $z \psi^{j, l}\left(0+v_{i}\right)$ tra-

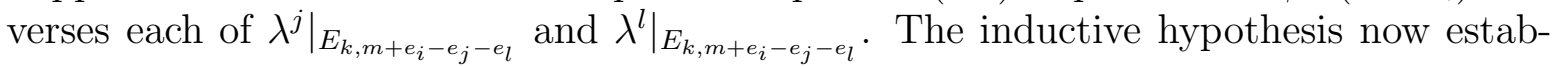
lishes Claim 2.

For $j \neq i$ such that $m_{j}>0$, let $\phi^{j}$ and $\lambda^{j}$ be as in Claim 1. Then Claim 2 implies that the formulae

$$
\left.\lambda_{x}\right|_{E_{k, m}}=\left.\lambda_{y}\right|_{E_{k, m}},\left.\quad \lambda_{x}\right|_{E_{k, m+e_{i}-e_{j}}}=\lambda^{j}, \quad \text { and }\left.\quad \lambda_{x}\right|_{E_{k,\left[m-e_{j}, m+e_{i}\right]}^{*}} ^{*}=\phi^{j}
$$

determine a well-defined coloured-graph morphism $\lambda_{x}: E_{k, m+e_{i}} \rightarrow E$. Moreover $\lambda_{x}$ is $\mathcal{C}$-compatible because each square occurring in $\lambda_{x}$ occurs in $\lambda_{y}$, in one of the $\lambda^{j}$ or in one of the $\phi^{j}$.

To see that $\lambda_{x}$ is the unique $\mathcal{C}$-compatible coloured-graph morphism which $x$ traverses, fix a $\mathcal{C}$-compatible coloured-graph morphism $\mu$ traversed by $x$. Then $y$ traverses $\left.\mu\right|_{E_{k, m}}$, so the inductive hypothesis implies that $\left.\mu\right|_{E_{k, m}}=\lambda_{y}$. Fix $j \neq i$ such that $m_{j}>0$. That $\mathcal{C}$ is a complete collection of squares and that $\lambda_{y}\left(\left(m-e_{j}\right)+v_{j}\right) f$ traverses $\left.\mu\right|_{E_{k,\left[m-e_{j}, m+e_{i}\right]}^{*}} ^{*}$ implies that $\left.\mu\right|_{E_{k,\left[m-e_{j}, m+e_{i}\right]}^{*}} ^{*}=\phi^{j}$. In particular, $\mu\left(\left(m-e_{j}\right)+v_{i}\right)=g^{j}$, and hence $z^{j} g^{j}$

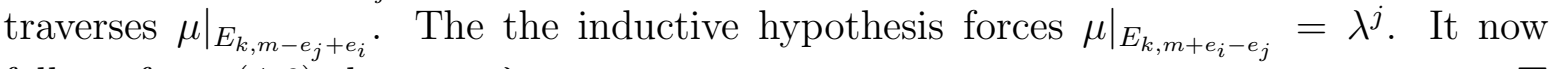
follows from (4.6) that $\mu=\lambda_{x}$.

Corollary 4.9. Let $E$ be a $k$-coloured graph and let $\mathcal{C}$ be a complete collection of squares in $E$ which is associative. If $\mu: E_{k, m} \rightarrow E$ and $\nu: E_{k, n} \rightarrow E$ are $\mathcal{C}$-compatible coloured-graph morphisms such that $s(\mu)=r(\nu)$, then there exists a unique $\mathcal{C}$-compatible 
coloured-graph morphism $\mu \nu: E_{k, m+n} \rightarrow E$, called the composition of $\mu$ and $\nu$ such that $\left.(\mu \nu)\right|_{E_{k, m}}=\mu$ and $\left.(\mu \nu)\right|_{E_{k,[m, m+n]}} ^{*}=\nu$.

Proof. Fix $x, y \in E^{*}$ such that $x$ traverses $\mu$ and $y$ traverses $\nu$. Proposition 4.7 implies that $x y$ traverses a unique $\mathcal{C}$-compatible coloured-graph morphism $\mu \nu$. Then $x$ traverses $\left.(\mu \nu)\right|_{E_{k, m}}$, and $y$ traverses $\left.(\mu \nu)\right|_{E_{k,[m, m+n]}} ^{*}$, so Proposition 4.7 implies that $\left.(\mu \nu)\right|_{E_{k, m}}=\mu$ and $\left.(\mu \nu)\right|_{E_{k,[m, m+n]}} ^{*}=\nu$.

Moreover, if $\lambda$ is any other coloured-graph morphism such that $\left.\lambda\right|_{E_{k, m}}=\mu$ and $\left.\lambda\right|_{E_{k,[m, m+n]}} ^{*}=\nu$ then Remark 3.2 shows that $x y$ traverses $\lambda$ so uniqueness in Proposition 4.7 forces $\lambda=\mu \nu$.

Remark 4.10. Let $E$ be a $k$-coloured graph and let $\mathcal{C}$ be a complete collection of squares in $E$ which is associative. Fix $m \leq n$ in $\mathbb{N}^{k}$ and suppose that $\lambda: E_{k, n} \rightarrow E$ is a $\mathcal{C}$-compatible coloured-graph morphism. Corollary 4.9 implies that $\mu:=\left.\lambda\right|_{E_{k, m}}$ and $\nu:=\left.\lambda\right|_{E_{k,[m, n]}} ^{*}$ satisfy $\mu \nu=\lambda$. Suppose that $\mu^{\prime}: E_{k, m} \rightarrow E$ and $\nu^{\prime}: E_{k, n-m} \rightarrow E$ are another two $\mathcal{C}$-compatible coloured-graph morphisms such that $\mu^{\prime} \nu^{\prime}=\lambda$. Then $\mu^{\prime}=\left.\lambda\right|_{E_{k, m}}=\mu$ and $\nu^{\prime}=\left.\lambda\right|_{E_{k,[m, n]}} ^{*}=\nu$. So $\mu$ and $\nu$ are the unique coloured-graph morphisms with $d(\mu)=m, d(\nu)=n$ and $\lambda=\mu \nu$.

Corollary 4.11. Let $E$ be a $k$-coloured graph and let $\mathcal{C}$ be a complete collection of squares in $E$ which is associative. If $\lambda: E_{k, l} \rightarrow E, \mu: E_{k, m} \rightarrow E$ and $\nu: E_{k, n} \rightarrow E$ are $\mathcal{C}$-compatible coloured-graph morphisms such that $s(\lambda)=r(\mu)$ and $s(\mu)=r(\nu)$, then $\lambda(\mu \nu)=(\lambda \mu) \nu$.

Proof. Fix $x_{\lambda}, x_{\mu}, x_{\nu} \in E^{*}$ such that $x_{\lambda}$ traverses $\lambda, x_{\mu}$ traverses $\mu$ and $x_{\nu}$ traverses $\nu$. Repeated applications of Remark 3.2 show that $x_{\lambda} x_{\mu}$ traverses $\lambda \mu$. Hence $x_{\lambda} x_{\mu} x_{\nu}=$ $\left(x_{\lambda} x_{\mu}\right) x_{\nu}$ traverses $(\lambda \mu) \nu$. Similarly, $x_{\lambda} x_{\mu} x_{\nu}=x_{\lambda}\left(x_{\mu} x_{\nu}\right)$ traverses $\lambda(\mu \nu)$. So Proposition 4.7 implies that $(\lambda \mu) \nu=\lambda(\mu \nu)$.

Proof of Theorem 4.4. The first statement of the theorem is precisely Corollary 4.9, We must check that $\Lambda$ is a category. For composable $\mu, \nu$ we have

$$
s(\mu \nu)=(\mu \nu)(d(\mu \nu))=\left.(\mu \nu)\right|_{E_{k,[d(\mu), d(\mu)+d(\nu)]}^{*}}(d(\nu))=\nu(d(\nu))=s(\nu),
$$

and similarly $r(\mu \nu)=r(\mu)$. Associativity of composition follows from Corollary 4.11, For $v \in E^{0}$, we have $r\left(\lambda_{v}\right)=\lambda_{v}(0)=v$ and $s\left(\lambda_{v}\right)=\lambda_{v}\left(d\left(\lambda_{v}\right)\right)=\lambda_{v}(0)=v$. Moreover, if $r(\mu)=\lambda_{v}$ and $s(\nu)=\lambda_{v}$, then Remark 4.10 implies that $\mu=\lambda_{v} \mu$ and $\nu=\nu \lambda_{v}$. Hence $\Lambda$ is a category.

Since $\mathbb{N}^{k}$ as a category has only one object, $d$ trivially respects $r$ and $s$. It follows immediately from the definition of composition (see Corollary 4.9) that $d$ respects composition. So $d$ is a functor. Remark 4.10 shows that $d$ satisfies the factorisation property. So $(\Lambda, d)$ is a $k$-graph.

It remains to show that $\rho$ defines an isomorphism of $E$ with $E_{\Lambda}$ and that $\rho \circ \phi \in \mathcal{C}_{\Lambda}$ for each $\phi \in \mathcal{C}$. The map $v \mapsto \overline{\lambda_{v}}$ is a bijection. We established above that $f \mapsto \lambda_{f}$ is a range- and source-preserving bijection between $c^{-1}\left(c_{i}\right) \subset E^{1}$ and $\Lambda^{e_{i}}$. We defined $E_{\Lambda}^{1}=\left\{\bar{f}: f \in \bigcup_{i=1}^{k} \Lambda^{e_{i}}\right\}$ (see Definition 4.1). For each $f \in E^{1}, \lambda_{f}$ is the unique coloured-graph morphism traversed by $f$, and $\overline{\lambda_{f}} \in E_{\Lambda}^{1}$ satisfies $c_{E_{\Lambda}}\left(\overline{\lambda_{f}}\right)=c_{i}=c(f)$, $r\left(\overline{\lambda_{f}}\right)=\overline{\lambda_{f}(0)}=\overline{\lambda_{r(f)}}$, and $s\left(\overline{\lambda_{f}}\right)=\overline{\lambda_{f}\left(e_{i}\right)}=\overline{\lambda_{s(f)}}$. Since $\rho^{1}$ is bijective, the pair 
$\left(\rho^{0}, \rho^{1}\right): E \rightarrow E_{\Lambda}$ is an isomorphism of coloured graphs. To see that it preserves squares, fix $\psi \in \mathcal{C}$. Then $\rho \circ \psi$ is the square $\phi_{\psi}$ of (4.1) and hence belongs to $\mathcal{C}_{\Lambda_{(E, \mathcal{C})}}$ as required.

Proof of Theorem 4.5. For $\gamma \in \Gamma$ define $\theta_{\gamma}: E_{k, m} \rightarrow E$ as in (4.3) and (4.4). Then $r\left(\theta_{\gamma}^{1}\left(m+v_{i}\right)\right)=r\left(\psi^{1}\left(\overline{\gamma\left(m, m+e_{i}\right)}\right)\right)=\psi^{0}(\gamma(m))=\theta_{\gamma}^{0}(m)$ and similarly at the source, so $\theta_{\gamma}$ is a graph morphism. Since $\psi^{1}$ preserves colour, we have

$$
c_{E}\left(\theta_{\gamma}^{1}\left(m+v_{i}\right)\right)=c_{E}\left(\psi^{1}\left(\overline{\gamma\left(m, m+e_{i}\right)}\right)\right)=c_{E_{\Gamma}}\left(\overline{\gamma\left(m, m+e_{i}\right)}\right)=c_{i}=c_{E_{k, d(\gamma)}}\left(m+v_{i}\right),
$$

so $\theta_{\gamma}$ is a coloured-graph morphism.

To see that $\theta_{\gamma}$ is $\mathcal{C}$-compatible, fix a square $\alpha$ occurring in $\theta_{\gamma}$. Then there exist $m \in \mathbb{N}^{k}$ and $i, j \leq k$ such that $\alpha(x)=\theta_{\gamma}(x+m)$ for all $x \in E_{k, e_{i}+e_{j}}$. Let $\lambda:=\gamma\left(m, m+e_{i}+e_{j}\right)$. Then $\alpha^{0}(n)=\theta_{\gamma}^{0}(m+n)=\psi^{0}(\lambda(n))$ for $0 \leq n \leq e_{i}+e_{j}$, and $\alpha^{1}\left(n+v_{l}\right)=\theta_{\gamma}^{1}\left(m+n+v_{l}\right)=$ $\psi^{1}\left(\overline{\lambda\left(n, n+e_{l}\right)}\right)$ whenever $n, n+e_{l} \leq e_{i}+e_{j}$. That is, $\alpha=\psi \circ \phi_{\lambda}$ where $\phi_{\lambda} \in \mathcal{C}_{\Gamma}$ is as in Definition 4.1. By hypothesis, that $\phi_{\lambda} \in \mathcal{C}_{\Gamma}$ implies that $\alpha \in \mathcal{C}$, and hence $\theta_{\gamma}$ is $\mathcal{C}$-compatible. Hence $\theta_{\gamma} \in \Lambda_{(E, \mathcal{C})}^{d(\gamma)}$.

The assignment $\gamma \mapsto \theta_{\gamma}$ is a degree, range and source preserving map $\theta: \Gamma \rightarrow \Lambda_{(E, \mathcal{C})}$. To see that $\theta$ is injective, fix $\gamma, \gamma^{\prime} \in \Gamma$ and suppose that $\theta_{\gamma}=\theta_{\gamma^{\prime}}$. Write $\gamma=\gamma_{1} \ldots \gamma_{n}$ where each $d\left(\gamma_{i}\right) \in\left\{e_{1}, \ldots, e_{k}\right\}$, and $\gamma^{\prime}=\gamma_{1}^{\prime} \ldots \gamma_{n}^{\prime}$ where each $d\left(\gamma_{i}^{\prime}\right)=d\left(\gamma_{i}\right)$. For $i \leq n$ define $p_{i}:=\sum_{j=1}^{i} d\left(\gamma_{j}\right)$. Then for each $i \leq n$,

$$
\psi^{1}\left(\overline{\gamma_{i}}\right)=\theta_{\gamma}^{1}\left(p_{i-1}, p_{i}\right)=\theta_{\gamma^{\prime}}^{1}\left(p_{i-1}, p_{i}\right)=\psi^{1}\left(\overline{\gamma_{i}^{\prime}}\right) .
$$

Since $\psi^{1}$ is injective, it follows that $\overline{\gamma_{i}}=\overline{\gamma_{i}^{\prime}}$ and hence $\gamma_{i}=\gamma_{i}^{\prime}$. So $\theta$ is injective.

To see that $\theta$ preserves composition, fix $\gamma, \gamma^{\prime} \in \Gamma$ with $s(\gamma)=r\left(\gamma^{\prime}\right)$, and fix paths $\psi^{1}\left(\overline{\gamma_{1}}\right) \ldots \psi^{1}\left(\overline{\gamma_{m}}\right)$ and $\psi^{1}\left(\overline{\gamma_{1}^{\prime}}\right) \ldots \psi^{1}\left(\overline{\gamma_{n}^{\prime}}\right)$ which traverse $\theta_{\gamma}$ and $\theta_{\gamma^{\prime}}$. Then

$$
\psi^{1}\left(\overline{\gamma_{1}}\right) \ldots \psi^{1}\left(\overline{\gamma_{m}}\right) \psi^{1}\left(\overline{\gamma_{1}^{\prime}}\right) \ldots \psi^{1}\left(\overline{\gamma_{n}^{\prime}}\right)
$$

traverses both $\theta_{\gamma \gamma^{\prime}}$ and $\theta_{\gamma} \theta_{\gamma^{\prime}}$. So $\theta_{\gamma \gamma^{\prime}}=\theta_{\gamma} \theta_{\gamma^{\prime}}$ by Proposition 4.7. So $\theta$ is a functor.

To see that $\theta$ is surjective, fix $\lambda \in \Lambda_{(E, \mathcal{C})}$ and a path $f_{1} \ldots f_{m}$ which traverses $\lambda$. Then each $f_{i} \in E^{1}$, and since $\psi^{1}$ is surjective, each $f_{i}=\psi^{1}\left(g_{i}\right)$ for some $g_{i} \in E_{\Gamma}^{1}$. Each $g_{i}=\overline{\gamma_{i}}$ for some $\gamma_{i} \in \Gamma$. Let $\gamma:=\gamma_{1} \ldots \gamma_{n}$. Then $f_{1} \ldots f_{m}=\psi^{1}\left(\overline{\gamma_{1}}\right) \ldots \psi^{1}\left(\overline{\gamma_{m}}\right)$ traverses both $\lambda$ and $\theta_{\gamma}$. So Proposition 4.7 implies that $\theta_{\gamma}=\lambda$ and hence $\theta$ is surjective. Thus $\theta$ is an isomorphism $\Gamma \cong \Lambda_{(E, \mathcal{C})}$.

Example 4.12. We now show how the 2-graphs of [18] fit our present mould. Let $(T, q, t, w)$ be basic data, as in [18, $\S 3]$. Thus $T$ is a finite hereditary subset of $\mathbb{N}^{2}$ with corners $d_{1} e_{1}, d_{2} e_{2}$ satisfying $d_{i} \geq 1, q \in \mathbb{N}, t \in \mathbb{Z} / q \mathbb{Z}$ and $w: T \rightarrow \mathbb{Z} / q \mathbb{Z}$ such that both $w\left(d_{1} e_{1}\right)$ and $w\left(d_{2} e_{2}\right)$ are nonzero. If $S$ is a subset of $\mathbb{N}^{2}$ which contains a translate $R+n$ of a set $R$ and $f: S \rightarrow \mathbb{Z} / q \mathbb{Z}$, then we define $\left.f\right|_{R+n} ^{*}$ to be the function $\left.f\right|_{R+n} ^{*}: R \rightarrow \mathbb{Z} / q \mathbb{Z}$ defined by $\left.f\right|_{R+n} ^{*}(i)=f(i+n)$. For $m \in \mathbb{N}^{2}$, we write $T(m):=\bigcup\{T+n: 0 \leq n \leq m\}$.

The vertices in the 2-graph $\Lambda(T, q, t, w)$ are the functions $v: T \rightarrow \mathbb{Z} / q \mathbb{Z}$ satisfying $\sum_{i \in T} w(i) v(i)=t$, the paths of degree $m$ are the functions $\lambda: T(m) \rightarrow \mathbb{Z} / q \mathbb{Z}$ such that $\left.\lambda\right|_{T+n} ^{*} \in \Lambda^{0}$ whenever $0 \leq n \leq m$, and the range and source of $\lambda \in \Lambda^{m}$ are given by $r(\lambda)=\left.\lambda\right|_{T}$ and $s(\lambda)=\left.\lambda\right|_{T+m} ^{*}$. It is shown in [18, Theorem 3.4] that there is a 
well-defined composition on $\Lambda(T, q, t, w)$ that makes $\Lambda(T, q, t, w)$ into a 2-graph with factorisations given by $\lambda=\left(\lambda_{T(n)}\right)\left(\left.\lambda\right|_{T(m-n)+n} ^{*}\right)$.

The 2-graph $\Lambda(T, q, t, w)$ fits our construction as follows. We define a coloured graph $E$ by $E^{0}=\Lambda^{0}, E^{c_{i}}:=\Lambda^{e_{i}}, E^{1}:=E^{c_{1}} \cup E^{c_{2}}$, and restricting $r, s$. Then for each path $\lambda \in \Lambda^{m}$, the formulas

$$
\mu_{\lambda}(n)=\left.\lambda\right|_{T+n} ^{*} \text { and } \mu_{\lambda}\left(n+v_{i}\right)=\left.\lambda\right|_{\left(T \cup\left(T+e_{i}\right)\right)+n} ^{*}
$$

define a coloured-graph morphism, and hence a path of degree $m$ in the 2-graph defined by $E$ with the squares given by the coloured-graph morphisms arising from the paths of degree $e_{i}+e_{j}$ in $\Lambda$. One can check, by directly constructing the inverse, that $\lambda \mapsto \mu_{\lambda}$ is an isomorphism of 2-graphs.

\section{TOPOLOGY OF PATH SPACES}

In [16. Proposition 4.3] the authors appeal to general category-theoretic results [23] to see that given a $k$-coloured graph $E$ and a complete collection of squares $\mathcal{C}$ in $E$ which is associative, the corresponding $k$-graph $\Lambda_{(E, \mathcal{C})}$ is isomorphic to the quotient of the category $E^{*}$ under the equivalence relation $\sim$ generated by

$$
\begin{aligned}
& \bigcup_{n \geq 2}\left\{(x, y) \in E^{n} \times E^{n}: \text { there exists } i<n\right. \text { such that } \\
& \left.\qquad x_{j}=y_{j} \text { whenever } j \notin\{i, i+1\} \text { and } x_{i} x_{i+1} \sim_{\mathcal{C}} y_{i} y_{i+1}\right\} .
\end{aligned}
$$

We start this section with a direct proof of this assertion by showing that each equivalence class for $\sim$ is the set of paths which traverse some $\lambda \in \Lambda_{(E, \mathcal{C})}$. We show that the quotient map extends to a surjection from the space of all paths in $E$ to the space of all paths in $\Lambda$.

We then restrict attention to $k$-graphs which are row-finite with no sources in the sense that $0<\left|v \Lambda^{e_{i}}\right|<\infty$ for all $v \in \Lambda^{0}$ and $i \leq k$ (see [14]). In this context, the space $\Lambda^{\infty}$ of infinite paths in $\Lambda$ (see Remark 5.3 for a precise definition) - under the topology with basic open sets $\mathcal{Z}(\mu):=\left\{x \in \Lambda^{\infty}: x(0, d(\mu))=\mu\right\}$ indexed by $\mu \in \Lambda$ - is a locally compact Hausdorff space. Furthermore, it is the unit space of the groupoid $\mathcal{G}_{\Lambda}$ used to define $C^{*}(\Lambda)$ in 14 .

We show that $\Lambda^{\infty}$ is the topological quotient of the space

$$
E^{k-\infty}:=\left\{x \in E^{\infty}:\left|\left\{i: c\left(x_{i}\right)=c_{j}\right\}\right|=\infty \text { for each } j \leq k\right\}
$$

of $k$-infinite paths in $E$. We also show that $E^{k-\infty}$ is a closed subspace of $E^{\infty}$. Lastly, we present an example which shows that these results do not necessarily hold if $\Lambda$ is not row-finite.

The following elementary lemma can be deduced from more general results in the literature (for example [10, Theorem 3.9]), but we provide a straightforward proof for completeness. Recall that $q$ denotes the quotient map from $\mathbb{F}_{k}^{+}$to $\mathbb{N}^{k}$.

Lemma 5.1. Fix $w, w^{\prime} \in \mathbb{F}_{k}^{+}$and suppose that $q(w)=q\left(w^{\prime}\right)$. Then there is a finite sequence $\left(w^{i}\right)_{i=1}^{m}$ in $\mathbb{F}_{k}^{+}$such that $w^{1}=w, w^{m}=w^{\prime}$, and for each $i<m$ there exists $j_{i}<|w|$ such that $w_{l}^{i}=w_{l}^{i+1}$ for $l \notin\left\{j_{i}, j_{i}+1\right\}, w_{j_{i}}^{i}=w_{j_{i}+1}^{i+1}$ and $w_{j_{i}+1}^{i}=w_{j_{i}}^{i+1}$. 
Proof. The result is trivial if $|w|=0$. Suppose $|w| \geq 1$ and the result holds for words of length $|w|-1$. Since $q(w)=q\left(w^{\prime}\right)$ there exists $j$ such that $w_{j}=w_{1}^{\prime}$. Let

$$
\begin{aligned}
w^{2} & =w_{1} \ldots w_{j-2} w_{j} w_{j-1} w_{j+1} \ldots w_{|w|}, \\
w^{3} & =w_{1} \ldots w_{j} w_{j-2} w_{j-1} w_{j+1} \ldots w_{|w|}, \\
& \vdots \\
w^{j} & =w_{j} w_{1} \ldots w_{j-2} w_{j-1} w_{j+1} \ldots w_{|w|} .
\end{aligned}
$$

Let $x=w_{1} \ldots w_{j-2} w_{j-1} w_{j+1} \ldots w_{|w|}$ and $x^{\prime}=w_{2}^{\prime} \ldots w_{|w|}^{\prime}$. Then $w^{j}=w_{1}^{\prime} x, w^{\prime}=w_{1}^{\prime} x^{\prime}$, $q(x)=q\left(x^{\prime}\right)$ and $|x|=|w|-1$. Apply the inductive hypothesis to $x$ and $x^{\prime}$ to obtain $x^{1}, \ldots, x^{n}$. The sequence $w^{1}, \ldots w^{j}, w_{1}^{\prime} x^{2}, \ldots, w_{1}^{\prime} x^{n}$ does the job.

Proposition 5.2. Let $E$ be a $k$-coloured graph and let $\mathcal{C}$ be a complete collection of squares in $E$ which is associative. Let $\sim$ be the equivalence relation on $E^{*}$ generated by (5.1). For $x, y \in E^{*}$, we have $x \sim y$ if and only if $x$ and $y$ traverse the same $\mathcal{C}$-compatible graph morphism $\lambda$. The structure maps $s([x]):=s(x), r([x]):=r(x)$, $d([x]):=q(c(x))$ and $[x][y]:=[x y]$ are well-defined on $E^{*} / \sim$, and under these operations $E^{*} / \sim$ is a k-graph which is isomorphic to $\Lambda_{(E, \mathcal{C})}$.

Proof. For a pair $(x, y)$ as in (5.1), we have $r(x)=r(y), s(x)=s(y)$, and $q(c(x))=$ $q(c(y))$, so the formulas $s([x]):=s(x), r([x]):=r(x)$ and $d([x]):=q(c(x))$ are welldefined.

If $x \sim y$, then there is a finite sequence of pairs $\left(x^{l}, x^{l+1}\right), 1 \leq l \leq m-1$, each of the form described in (5.1) such that $x^{1}=x$ and $x^{m}=y$. So it suffices to fix $(x, y)$ as in (5.1) and show that $x$ and $y$ traverse the same $\mathcal{C}$-compatible coloured-graph morphism. For this, let $\phi$ be the square in $\mathcal{C}$ traversed by $x_{i} x_{i+1}$ and hence also by $y_{i} y_{i+1}$. By Proposition 4.7, $x_{1} \ldots x_{i-1}=y_{1} \ldots y_{i-1}$ traverses a unique $\mathcal{C}$-compatible morphism $\mu$ and $x_{i+2} \ldots x_{n}=y_{i+2} \ldots y_{n}$ traverses a unique $\mathcal{C}$-compatible morphism $\nu$. By Corollary 4.9, there is a unique $\mathcal{C}$-compatible $\lambda=\mu \phi \nu$ which agrees, upon restriction, with each of $\mu, \phi$ and $\nu$. Each of $x$ and $y$ traverse this $\lambda$.

Now suppose that $x$ and $y$ traverse a common $\mathcal{C}$-compatible morphism $\lambda$. Then in particular $q(c(x))=q(c(y))$. By Lemma 5.1 there is a finite sequence $\left(w^{i}\right)_{i=1}^{m}$ in $\mathbb{F}_{k}^{+}$ such that $w^{1}=c(x), w^{m}=c(y)$, and for each $i \leq m-1$ there exists $j_{i}<|x|$ such that $w_{l}^{i}=w_{l}^{i+1}$ for $l \notin\left\{j_{i}, j_{i}+1\right\}$, and $w_{j_{i}}^{i}=w_{j_{i}+1}^{i+1}$ and $w_{j_{i}+1}^{i}=w_{j_{i}}^{i+1}$. For each $i$, let $z^{i}$ be the path which traverses $\lambda$ such that $c\left(x^{i}\right)=w^{i}$, and for each $i \leq m$ and $l \leq|x|$, let $p_{l}^{i}:=q\left(c\left(x_{1}^{i} \cdots x_{l}^{i}\right)\right)$. Then for each $i \leq m$, both $x_{1}^{i} \ldots x_{j_{i}-1}^{i}$ and $x_{1}^{i+1} \ldots x_{j_{i}-1}^{i+1}$ traverse $\left.\lambda\left(0, p_{j_{i}-1}^{i}\right)\right)$, so they are equal, and likewise $x_{j_{i}+2}^{i} \ldots x_{|x|}^{i}=x_{j_{i}+2}^{i+1} \ldots x_{|x|}^{i+1}$. Moreover each of $x_{j_{i}}^{i} x_{j_{i}+1}^{i}$ and $x_{j_{i}}^{i+1} x_{j_{i}+1}^{i+1}$ traverses $\left.\lambda\right|_{\left[p_{j_{i}-1}^{i}, p_{j_{i}+1}^{i}\right.} ^{*}$, which, since $\lambda$ is $\mathcal{C}$-compatible, belongs to $\mathcal{C}$. Thus the pair $\left(x^{i}, x^{i+1}\right)$ is a pair of paths as in (5.1), and it follows that $x \sim y$ as required.

By the preceding two paragraphs, the assignment $\rho: \lambda \mapsto[x]$ for any $x$ which traverses $\lambda$ is a well-defined bijection from $\Lambda_{(E, \mathcal{C})}$ to $E^{*} / \sim$ which preserves range, source and degree. By definition of composition in $\Lambda_{(E, \mathcal{C})}$, if $x$ traverses $\mu$ and $y$ traverses $\mu$, then $x y$ traverses $\lambda \mu$. So if $[x]=\left[x^{\prime}\right]$ and $[y]=\left[y^{\prime}\right]$, then $x$ and $x^{\prime}$ both traverse $\mu$, and $y$ and 
$y^{\prime}$ both traverse $\nu$, so $x y$ and $x^{\prime} y^{\prime}$ both traverse $\mu \nu$. Thus

$$
[x y]=\rho(\mu \nu)=\left[x^{\prime} y^{\prime}\right],
$$

showing that the composition on $E^{*} / \sim$ is well-defined. So $\rho$ is a degree-preserving bijective functor, and hence an isomorphism of $k$-graphs.

We recall the $k$-graphs $\Omega_{k, m}$ described in [19, Examples 2.2]. For $m \in(\mathbb{N} \cup\{\infty\})^{k}$, define $\Omega_{k, m}$ be the category with $\operatorname{Obj}\left(\Omega_{k, m}\right)=\left\{n \in \mathbb{N}^{k}: n \leq m\right\}, \operatorname{Mor}\left(\Omega_{k, m}\right)=$ $\left\{(p, q) \in \mathbb{N}^{k} \times \mathbb{N}^{k}: p \leq q \leq m\right\}, s(p, q)=q, r(p, q)=p$ and $(p, q)(q, r)=(p, r)$. Then with $d(p, q)=q-p$, the pair $\left(\Omega_{k, m}, d\right)$ is a row-finite $k$-graph. By convention, $\Omega_{k}=\Omega_{k,(\infty, \ldots, \infty)}$. Note that there is only one possible complete collection of squares $\mathcal{C}$ in the $k$-coloured graph $E_{k, m}$, this collection is also associative, and the $k$-graph $\Lambda_{E_{k, m}, \mathcal{C}}$ of Theorem 4.4 is isomorphic to $\Omega_{k, m}$.

Remark 5.3. Let $\Lambda$ be a $k$-graph. For $m \in \mathbb{N}^{k}$, the factorisation property gives a bijection $\lambda \mapsto x_{\lambda}$ between $\Lambda^{m}$ and the set of graph morphisms from $\Omega_{k, m}$ to $\Lambda$ : for $\lambda \in \Lambda$ and $p \leq q \leq d(\lambda), x_{\lambda}(p, q)$ is the unique element of $\Lambda^{q-p}$ such that $\lambda=\lambda^{\prime} x_{\lambda}(p, q) \lambda^{\prime \prime}$ for some $\lambda^{\prime}, \lambda^{\prime \prime}$. By analogy, for $m \in(\mathbb{N} \cup\{\infty\})^{k}$, we call a $k$-graph morphism $x: \Omega_{k, m} \rightarrow \Lambda$ a path of degree $m$ in $\Lambda$, and we write $d(x)$ for $m$ and $r(x)$ for $x(0)$. We continue to denote the collection of all such paths by $\Lambda^{m}$. It is conventional to identify $\lambda$ with $x_{\lambda}$, and in particular to denote $x_{\lambda}(p, q)$ by $\lambda(p, q)$; so $\lambda=\lambda(0, p) \lambda(p, q) \lambda(q, d(\lambda))$ whenever $0 \leq p \leq q \leq d(\lambda)$

We shall write $W_{\Lambda}$ for the path space $W_{\Lambda}:=\bigcup_{m \in(\mathbb{N} \cup\{\infty\})^{k}} \Lambda^{m}$ of $\Lambda$.

Proposition 5.4 (cf. [14, Remarks 2.2]). Let $E$ be a $k$-coloured graph and let $\mathcal{C}$ be a complete collection of squares in $E$ which is associative. The map $x \mapsto \lambda_{x}$ from $E^{*}$ to $\Lambda=\Lambda_{(E, \mathcal{C})}$ of Proposition 4.7 extends uniquely to a degree-preserving map $\pi: W_{E} \rightarrow W_{\Lambda}$ such that for $x \in W_{E}$ and $i \in \mathbb{N}$ with $i \leq|x|, \pi(x)\left(0, d\left(x_{1} \ldots x_{i}\right)\right)=\lambda_{x_{1} \ldots x_{i}}$. Moreover, $\pi$ is surjective.

Remark 5.5. We have used the same symbol $\pi$ both for the map from $W_{E}$ to $W_{\Lambda}$ of Proposition 5.4, and for the map from $E_{\Lambda}$ to $\Lambda$ of Definition 4.1. This notation is consistent because Theorem 4.4 yields a coloured-graph isomorphism $E \cong E_{\Lambda}$ which carries elements of $\mathcal{C}$ to elements of $\mathcal{C}_{\Lambda}$.

Proof of Proposition 5.4. For $x \in W_{E}$ and $m \leq n \leq d(x)$, let $j$ be the least element of $\mathbb{N}$ such that $d\left(x_{1} \ldots x_{j}\right) \geq n$, and define $\pi(x)(m, n):=\left.\lambda_{x_{1} \ldots x_{j}}\right|_{E_{k,[m, n]}} ^{*}$. Proposition 4.7 implies that for $j \leq l$, we have $\left.\lambda_{x_{1} \ldots x_{l}}\right|_{E_{k, d\left(x_{1} \ldots x_{j}\right)}}=\lambda_{x_{1} \ldots x_{j}}$. Hence $\pi(x)\left(0, d\left(x_{1} \ldots x_{j}\right)\right)=$ $\lambda_{x_{1} \ldots x_{j}}$ for all $j \leq|x|$. The factorisation property in $\Lambda$ implies that $\pi(x)$ is a $k$-graph morphism from $\Omega_{k, d(x)}$ to $\Lambda$. For uniqueness of $\pi$, observe that by uniqueness of factorisations in $\Lambda$, any $y \in W_{\Lambda}$ such that $y\left(x_{1} \ldots x_{i}\right)=\lambda_{x_{1} \ldots x_{i}}$ for all $i \leq d(x)$ must satisfy $y(m, n)=\left.\lambda_{x_{1} \ldots x_{j}}\right|_{E_{k,[m, n]}} ^{*}$ whenever $d\left(x_{1} \ldots x_{j}\right) \geq n$.

To see that $\pi$ is surjective first note that if $\lambda \in \Lambda$ then any path $x$ which traverses $\lambda$ satisfies $\pi(x)=\lambda$. So fix $y \in W_{\Lambda} \backslash \Lambda$. Fix a sequence $\left(m_{j}\right)_{j=0}^{\infty}$ such that $m_{0}=0$, $m_{j+1}-m_{j} \in\left\{e_{1}, \ldots, e_{k}\right\}$ for all $j$ and $\bigvee_{j \in \mathbb{N}} m_{j}=d(y)$. For each $j \in \mathbb{N}$ define $x_{j}:=$ $y\left(m_{j-1}, m_{j}\right) \in E^{1}$. Then $x=x_{1} x_{2} \cdots \in W_{E}$, and $\pi(x)(m, n)=y(m, n)$ for all $m, n$ by uniqueness of factorisations in $\Lambda$, so $\pi(x)=y$. 
If $\pi: W_{E} \rightarrow W_{\Lambda}$ is the surjection of Proposition 5.4, then Proposition 4.9 implies that $\pi(x) \pi(y)=\pi(x y)$ when $x$ and $y$ are finite with $r(y)=s(x)$.

Now let $\Lambda$ be a row-finite $k$-graph with no sources. Recall that $\Lambda^{\infty}$ is the collection of $k$-graph morphisms from $\Omega_{k}$ to $\Lambda$, and $E^{k-\infty}$ is the collection of infinite paths in $E$ which contain infinitely many edges of each colour. Since $\pi$ is surjective, it follows that $\Lambda^{\infty}=\pi\left(E^{k-\infty}\right)$.

Remark 5.6. Let $E$ be a $k$-coloured graph and let $\mathcal{C}$ be a complete collection of squares in $E$ which is associative. Let $\Lambda=\Lambda_{(E, \mathcal{C})}$ be the corresponding $k$-graph as in Theorem 4.4. Identify $\Lambda^{0}$ with $E^{0}$. Then for each $v \in \Lambda^{0}$ and $i \leq k$, we have $\left|v \Lambda^{e_{i}}\right|=\mid\left\{e \in E^{1}\right.$ : $r(e)=v$ and $\left.c(e)=c_{i}\right\} \mid$. Hence $\Lambda$ is row-finite and has no sources if and only if $0<\mid\left\{e \in E^{1}: r(e)=v\right.$ and $\left.c(e)=c_{i}\right\} \mid<\infty$ for all $v \in E^{0}$ and $i \leq k$.

Proposition 5.7. Let $E$ be a $k$-coloured graph and let $\mathcal{C}$ be a complete collection of squares in $E$ which is associative. Let $\Lambda=\Lambda_{(E, \mathcal{C})}$ as in Theorem 4.4. Suppose that $\Lambda$ is row-finite and has no sources. Let $\pi: E^{k-\infty} \rightarrow \Lambda^{\infty}$ be the restriction of the surjection of Proposition 5.4. Then $U \subseteq \Lambda^{\infty}$ is open if and only if $\pi^{-1}(U) \subseteq E^{k-\infty}$ is open.

Proof. First suppose that $U$ is open in $\Lambda^{\infty}$, and fix $x \in \pi^{-1}(U)$. We seek a basic open set $B_{x}$ in $E^{k-\infty}$ such that $x \in B_{x} \subset \pi^{-1}(U)$. Since $U$ is open, there exists $\mu \in \Lambda$ such that $\pi(x) \in \mathcal{Z}(\mu) \subset U$. Fix $n \in \mathbb{N}$ such that $q\left(c\left(x_{1} \ldots x_{n}\right)\right)>d(\mu)$. Then $\pi\left(x_{1} \ldots x_{n}\right) \in \mathcal{Z}(\mu)$. Let $y_{x}=x_{1} \ldots x_{n}$. Then $x \in \mathcal{Z}\left(y_{x}\right)$. To see $\mathcal{Z}\left(y_{x}\right) \subset \pi^{-1}(U)$, fix $y \in \mathcal{Z}\left(y_{x}\right)$; say $y=y_{x} y^{\prime}$. Then $\pi(y)=\pi\left(x_{1} \ldots x_{n} y^{\prime}\right)=\pi\left(x_{1} \ldots x_{n}\right) \pi\left(y^{\prime}\right) \in \mathcal{Z}(\mu) \subset U$, so $y \in \pi^{-1}(U)$ as required.

For the reverse implication, suppose that $\pi^{-1}(U)$ is open in $E^{k-\infty}$, and fix $\lambda \in U$. We seek a basic open set $B_{\lambda}$ such that $\lambda \in B_{\lambda} \subset U$. Fix $x \in E^{\infty}$ which traverses $\lambda$. Then $x \in E^{k-\infty}$, and $x \in \pi^{-1}(U)$ which is open. Hence there exists a basic open set $B_{x} \in E^{k-\infty}$ such that $x \in B_{x} \subset \pi^{-1}(U)$. So $B_{x}=\mathcal{Z}\left(y_{x}\right)$ for some $y_{x} \in E^{*}$, and

$$
\lambda=\pi(x)=\pi\left(y_{x} x^{\prime}\right)=\pi\left(y_{x}\right) \pi\left(x^{\prime}\right) \in \mathcal{Z}\left(\pi\left(y_{x}\right)\right) .
$$

To see that $\mathcal{Z}\left(\pi\left(y_{x}\right)\right) \subset U$, let $\mu \in \mathcal{Z}\left(\pi\left(y_{x}\right)\right)$. Write $\mu=\pi\left(y_{x}\right) \mu^{\prime}$, and let $x_{\mu^{\prime}}$ be a path in $E^{*}$ which traverses $\mu^{\prime}$. Then $y_{x} x_{\mu^{\prime}} \in \mathcal{Z}\left(y_{x}\right) \subset \pi^{-1}(U)$, which implies that $\mu=\pi\left(y_{x} x_{\mu^{\prime}}\right) \in U$.

Proposition 5.7 implies that when $E$ is row-finite, the topology on $\Lambda^{\infty}$ is the quotient topology inherited from $E^{k-\infty}$ under $\pi$. In particular, $\pi$ is continuous.

As mentioned in the opening of this section, when $\Lambda$ is row-finite with no sources, $\Lambda^{\infty}$ is homeomorphic to the unit space of the groupoid $\mathcal{G}_{\Lambda}$ of [14]; it is also homeomorphic to the spectrum of the commutative subalgebra of $C^{*}(\Lambda)$ spanned by the projections $s_{\lambda} s_{\lambda}^{*}$ (see [26] and the opening of Section 6). If $\Lambda$ is not row-finite, this is no longer the case: $\Lambda^{\infty}$ need not be locally compact. To see this, suppose that $\Lambda$ is the 1 -graph with one vertex and infinitely many edges $\left\{f_{i}: i \in \mathbb{N}\right\}$. Then given any $x \in \Lambda^{\infty}$, any neighbourhood of $x$ contains $\mathcal{Z}\left(x_{1} \ldots x_{n}\right)$ for some $n$, and the cover $\mathcal{Z}\left(x_{1} \ldots x_{n}\right)=\bigcup_{i=1}^{\infty} \mathcal{Z}\left(x_{1} \ldots x_{n} f_{i}\right)$ has no finite subcover.

Instead, given a finitely aligned $k$-graph, let

$$
\begin{aligned}
\Lambda^{\leq \infty}:=\left\{x \in W_{\Lambda}\right. & \text { there exists } n \leq d(x) \text { such that } \\
& \left.\left(n \leq p \leq d(x) \text { and } p_{i}=d(x)_{i}\right) \text { implies } x(p) \Lambda^{e_{i}}=\emptyset\right\}
\end{aligned}
$$


as in [20]. Endow $W_{\Lambda}$ with the topology with basic open sets $\mathcal{Z}(\mu \backslash G):=\mathcal{Z}(\mu) \backslash$ $\left(\bigcup_{\lambda \in G} \mathcal{Z}(\mu \lambda)\right)$, where $\mu$ ranges over $\Lambda$ and $G$ ranges over all finite subsets of $s(\mu) \Lambda$. Then the unit space of $\partial \Lambda$ of the groupoid $\mathcal{G}_{\Lambda}$ constructed in [8] is the closure of $\Lambda \leq \infty$ in $W_{\Lambda}$; this is also homeomorphic to the spectrum of $\overline{\operatorname{span}}\left\{s_{\lambda} s_{\lambda}^{*}: \lambda \in \Lambda\right\}$ [26]. So the natural question to ask for finitely aligned $k$-graphs is whether the quotient topology on $\partial \Lambda$ is the same as its standard topology. The next example shows that it is not.

Example 5.8. Let $E$ be the 2-coloured graph pictured below.

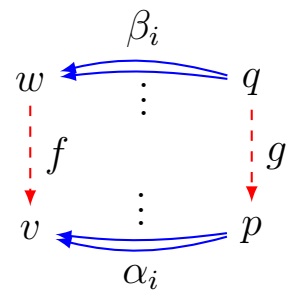

Let $\mathcal{C}$ be the collection of graph morphisms $\lambda_{i}: E_{2,(1,1)} \rightarrow E$ such that $\alpha_{i} g$ and $f \beta_{i}$ both traverse $\lambda_{i}$ for each $i$. This is a complete collection of squares in $E$. Since $E$ has only two colours $\mathcal{C}$ is associative. Let $\Lambda$ be the 2-graph constructed from $(E, \mathcal{C})$ as in Theorem 4.4, and let $\pi: W_{E} \rightarrow W_{\Lambda}$ be the surjection of Proposition 5.4. Then $v \Lambda^{\leq \infty}=\Lambda^{(1,1)}=\left\{\lambda_{i}: i \in \mathbb{N}\right\}$ and $\pi\left(\alpha_{i} g\right)=\lambda_{i}=\pi\left(f \beta_{i}\right)$ for all $i$.

We claim that $\alpha_{i} g \rightarrow v$ in $W_{E}$ but that $\lambda_{i} \rightarrow f$ in $W_{\Lambda}$. To see that $\alpha_{i} g \rightarrow v$ in $W_{E}$, fix a basic open set $\mathcal{Z}(y \backslash F) \subset E^{*}$ containing $v$. Then $y=v$. Since $F$ is finite, there are only finitely many $i$ such that either $\alpha_{i}$ or $\alpha_{i} g$ belongs to $F$. Let $N_{0}:=\max \left\{i: \alpha_{i} \in F\right.$ or $\left.\alpha_{i} g \in F\right\}$. Then $\alpha_{n} g \in \mathcal{Z}(v \backslash F)$ for all $n \geq N_{0}$, whence $\alpha_{i} g \rightarrow v$ as $i \rightarrow \infty$.

To see that $\lambda_{i} \rightarrow \pi(f)$ in $W_{\Lambda}$, fix a basic open set $\mathcal{Z}(\mu \backslash G) \subset \Lambda$ containing $f$. Then either $\mu=\pi(f)$ or $\mu=v$. We show that $\lambda_{i} \in \mathcal{Z}(\mu \backslash G)$ for large $i$. First suppose that $\mu=\pi(f)$. Then $G$ is a finite collection of paths of the form $\pi\left(\beta_{i} \nu\right)$. Let $N_{1}=\max \left\{i: \pi\left(\beta_{i} \nu\right) \in G\right.$ for some $\left.\nu\right\}$. Then $\lambda_{n}=\pi\left(f \beta_{n}\right) \in \mathcal{Z}(f \backslash G)$ for all $n \geq N_{1}$. Now suppose that $\mu=v$. Since $G$ does not contain $\pi(f)$, it is a finite subset of $\left\{\pi\left(\alpha_{i}\right), \lambda_{i}: i \in \mathbb{N}\right\}$. Let $N_{2}=\max \left\{i: \pi\left(\alpha_{i}\right) \in G\right.$ or $\left.\lambda_{i} \in G\right\}$. Then $\lambda_{n} \in \mathcal{Z}(v \backslash G)$ for all $n \geq N_{2}$. Hence $\lambda_{i} \rightarrow f$ as $i \rightarrow \infty$.

We now have $\pi\left(\lim \alpha_{i} g\right)=\pi(v) \neq \pi(f)=\lim \lambda_{i}=\lim \pi\left(\alpha_{i} g\right)$, so $\pi$ is not continuous.

\section{Simplicity of $C^{*}$-AlgeBras OF HigheR-RANK GRAPHS}

Suppose that $\Lambda$ is a $k$-graph which is row-finite and has no sources. For such $\Lambda$, a Cuntz-Krieger $\Lambda$-family in a $C^{*}$-algebra $B$ consists of partial isometries $\left\{t_{\lambda}: \lambda \in \Lambda\right\}$ satisfying the Cuntz-Krieger relations [14]:

(CK1) $\left\{t_{v}: v \in \Lambda^{0}\right\}$ are mutually orthogonal projections;

(CK2) $t_{\lambda \mu}=t_{\lambda} t_{\mu}$ for all $\lambda, \mu \in \Lambda$ with $s(\lambda)=r(\mu)$;

(CK3) $t_{\lambda}^{*} t_{\lambda}=t_{s(\lambda)}$ for all $\lambda \in \Lambda$; and

(CK4) $t_{v}=\sum_{\lambda \in v \Lambda^{m}} t_{\lambda} t_{\lambda}^{*}$ for all $v \in \Lambda^{0}$ and $m \in \mathbb{N}^{k}$.

The graph $C^{*}$-algebra $C^{*}(\Lambda)$ is the $C^{*}$-algebra generated by a universal Cuntz-Krieger $\Lambda$-family $\left\{s_{\lambda}: \lambda \in \Lambda\right\}$; it follows from [14, Proposition 2.11] that each vertex projection $s_{v}$ is nonzero. 
As in [21], we say that $\Lambda$ is aperiodic if for every vertex $v \in \Lambda^{0}$ and each pair $m \neq n \in \mathbb{N}^{k}$ there is a path $\lambda \in v \Lambda$ such that $d(\lambda) \geqslant m \vee n$ and

$$
\lambda(m, m+d(\lambda)-(m \vee n)) \neq \lambda(n, n+d(\lambda)-(m \vee n)) .
$$

Lemma 3.2 of [21] implies that this formulation of aperiodicity in terms of finite paths is equivalent to the aperiodicity condition used in [14]. So the next theorem follows from [14, Theorem 4.6].

Theorem 6.1 (The Cuntz-Krieger uniqueness theorem). Let $\Lambda$ be a row-finite, aperiodic $k$-graph with no sources. Suppose that $\left\{t_{\lambda}: \lambda \in \Lambda\right\}$ is a Cuntz-Krieger $\Lambda$-family, and let $\pi$ be the homomorphism of $C^{*}(\Lambda)$ such that $\pi\left(s_{\lambda}\right)=t_{\lambda}$ for all $\lambda \in \Lambda$. If each $t_{v}$ is nonzero, then $\pi$ is faithful.

The proof of this theorem in [14] uses a groupoid model for $C^{*}(\Lambda)$. Here we outline a direct proof that flows from the finite-path formulation of aperiodicity via the following lemma.

Lemma 6.2. Let $(\Lambda, d)$ be an aperiodic $k$-graph with no sources. Suppose that $v \in \Lambda^{0}$ and $l \in \mathbb{N}^{k}$. Then there exists $\lambda \in \Lambda$ such that $r(\lambda)=v, d(\lambda) \geq l$ and

$$
\alpha, \beta \in \Lambda v, d(\alpha), d(\beta) \leq l \text { and } \alpha \neq \beta \Longrightarrow(\alpha \lambda)(0, d(\lambda)) \neq(\beta \lambda)(0, d(\lambda)) .
$$

Proof. We list pairs $(m, n)$ of distinct elements of $\mathbb{N}^{k}$ with $0 \leq m, n \leq l$ as $\left\{\left(m^{(i)}, n^{(i)}\right)\right.$ : $1 \leq i \leq p\}$. Then an induction on $i$ shows that there exist $\mu_{i}$ and $l^{(i)} \in \mathbb{N}^{k}$ such that $r\left(\mu_{1}\right)=v, r\left(\mu_{i}\right)=s\left(\mu_{i-1}\right)$ for $i \geq 1, d\left(\mu_{i}\right)=\left(m^{(i)} \vee n^{(i)}\right)+l^{(i)}$, and $\mu_{i}\left(m^{(i)}, m^{(i)}+l^{(i)}\right) \neq$ $\mu_{i}\left(n^{(i)}, n^{(i)}+l^{(i)}\right)$. We now choose an arbitrary path $\lambda^{\prime}$ with $d\left(\lambda^{\prime}\right) \geq l$ and $r\left(\lambda^{\prime}\right)=s\left(\mu_{p}\right)$, and claim that $\lambda:=\mu_{1} \mu_{2} \cdots \mu_{p} \lambda^{\prime}$ has the required properties. We trivially have $d(\lambda) \geq l$.

Suppose that $\alpha$ and $\beta$ are distinct paths with source $v$ and $d(\alpha) \vee d(\beta) \leq l$. If $d(\alpha)=d(\beta)=d$, say, then the initial segments $\alpha=(\alpha \lambda)(0, d)$ and $\beta=(\beta \lambda)(0, d)$ are not equal, and $\alpha \lambda \neq \beta \lambda$. So suppose that $d(\alpha) \neq d(\beta)$, say $(d(\alpha), d(\beta))=\left(m^{(i)}, n^{(i)}\right)$. Let $d:=\sum_{j=1}^{i-1} d\left(\mu_{j}\right)$. Then

$$
(\alpha \lambda)\left(d(\alpha)+d+n^{(i)}, d(\alpha)+d+n^{(i)}+l^{(i)}\right)=\mu_{i}\left(n^{(i)}, n^{(i)}+l^{(i)}\right)
$$

is not the same as $\mu_{i}\left(m^{(i)}, m^{(i)}+l^{(i)}\right)=(\beta \lambda)\left(d(\beta)+d+m^{(i)}, d(\beta) d+m^{(i)}+l^{(i)}\right)$. Since $d(\beta)+d+m^{(i)}=d+m^{(i)}+n^{(i)}=d(\alpha)+d+n^{(i)}$, it follows that

$$
(\alpha \lambda)\left(d+m^{(i)}+n^{(i)}, d+m^{(i)}+n^{(i)}+l^{(i)}\right) \neq(\beta \lambda)\left(d+m^{(i)}+n^{(i)}, d+m^{(i)}+n^{(i)}+l^{(i)}\right) .
$$

The presence of the factor $\lambda^{\prime}$ forces $d(\lambda) \geq d+m^{(i)}+n^{(i)}+l^{(i)}$, so $(\alpha \lambda)(0, d(\lambda)) \neq$ $(\beta \lambda)(0, d(\lambda))$, as required.

Proposition 6.3. Suppose that $\Lambda$ is a row-finite aperiodic $k$-graph with no sources, and let $\left\{t_{\lambda}: \lambda \in \Lambda\right\}$ be a Cuntz-Krieger $\Lambda$-family in a $C^{*}$-algebra $B$ such that $t_{v} \neq 0$ for all $v \in \Lambda^{0}$. Let $F$ be a finite subset of $\Lambda$ and let $a:(\mu, \nu) \mapsto a_{\mu, \nu}$ be $a \mathbb{C}$-valued function on $F \times F$ such that $s(\mu)=s(\nu)$ whenever $a_{\mu, \nu} \neq 0$. Then

$$
\left\|\sum_{\mu, \nu \in F} a_{\mu, \nu} t_{\mu} t_{\nu}^{*}\right\| \geq\left\|\sum_{\mu, \nu \in F, d(\mu)=d(\nu)} a_{\mu, \nu} t_{\mu} t_{\nu}^{*}\right\| .
$$


Proof. Let $a:=\sum_{\mu, \nu \in F} a_{\mu, \nu} t_{\mu} t_{\nu}^{*}$ and let $a_{0}:=\sum_{\mu, \nu \in F, d(\mu)=d(\nu)} a_{\mu, \nu} t_{\mu} t_{\nu}^{*}$. Define $n:=$ $\bigvee_{\mu \in F} d(\mu)$, and let $G:=\bigcup_{\mu \in F} F s(\mu) \Lambda^{n-d(\mu)}$. So if $\mu, \nu \in F$ with $s(\mu)=s(\nu)$ and $d(\mu \alpha)=n$, then $\mu \alpha, \nu \alpha \in G$. By applying (CK4) at $s(\mu)$ for each $\mu, \nu \in F$, we can express

$$
a=\sum_{\mu, \nu \in G} b_{\mu, \nu} t_{\mu} t_{\nu}^{*} \quad \text { and } \quad a_{0}=\sum_{\mu, \nu \in G, d(\mu)=d(\nu)} b_{\mu, \nu} t_{\mu} t_{\nu}^{*}
$$

where $b_{\mu, \nu} \neq 0$ implies $d(\mu)=n$ and $s(\mu)=s(\nu)$.

For each $v \in s(G)$, apply Lemma 6.2 with $l=\bigvee_{\nu \in G} d(\nu)$ to find $\lambda_{v} \in v \Lambda$ such that $d(\lambda) \geq l$ and

$$
\left(\alpha \lambda_{v}\right)(0, l) \neq\left(\beta \lambda_{v}\right)(0, l) \text { for distinct } \alpha, \beta \in G v,
$$

and let $Q_{v}:=\sum_{\alpha \in G v, d(\alpha)=n} t_{\alpha \lambda_{v}} t_{\alpha \lambda_{v}}^{*}$. Then (CK3) implies that the $Q_{v}$ are mutually orthogonal projections. Hence

$$
\left\|\sum_{v \in s(G)} Q_{v} a Q_{v}\right\| \leq\|a\| .
$$

We show that

$$
\sum_{v \in s(G)} Q_{v} a Q_{v}=\sum_{v \in s(G)} Q_{v} a_{0} Q_{v}
$$

For $\mu, \nu \in G$ with $s(\mu)=s(\nu)$ and $d(\mu)=n$, a quick calculation using (CK4) gives

$$
Q_{v} t_{\mu} t_{\nu}^{*}=\delta_{v, s(\mu)} t_{\mu \lambda_{s(\mu)}} t_{\nu \lambda_{s(\mu)}}^{*}
$$

Suppose $d(\mu) \neq n$ and fix $\alpha \in G \cap \Lambda^{n}$. Then $\left(\alpha \lambda_{s(\alpha)}\right)(0, l) \neq\left(\nu \lambda_{s(\nu)}\right)(0, l)$, and hence $t_{\nu \lambda_{s(\mu)}}^{*} t_{\alpha \lambda_{s(\alpha)}}=0$. This and (6.4) give $Q_{v} t_{\mu} t_{\nu}^{*} Q_{v}=0$ for all $v$, and (6.3) follows.

Finally we show that

$$
\left\|\sum_{v \in s(G)} Q_{v} a_{0} Q_{v}\right\|=\left\|a_{0}\right\| .
$$

Routine calculations using the Cuntz-Krieger relations and that the $t_{v}$ are all nonzero show that $\left\{t_{\mu} t_{\nu}^{*}: \mu, \nu \in G \cap \Lambda^{n}, s(\mu)=s(\nu)\right\}$ is a family of nonzero matrix units spanning an isomorphic copy of $\bigoplus_{v \in s(G)} M_{G v \cap \Lambda^{n}}(\mathbb{C})$, and that $\left\{t_{\mu \lambda_{s(\mu)}} t_{\nu \lambda_{s(\nu)}}: \mu, \nu \in G \cap \Lambda^{n}, s(\mu)=\right.$ $s(\nu)\}$ is a family of nonzero matrix units for the same finite-dimensional $C^{*}$-algebra. Hence $t_{\mu} t_{\nu}^{*} \mapsto t_{\mu \lambda_{s(\mu)}} t_{\nu \lambda_{s(\nu)}}^{*}$ determines an isomorphism of finite-dimensional subalgebras of $C^{*}(\Lambda)$, so is isometric. Calculations like (6.4) show that $\sum_{v \in s(G)} Q_{v} t_{\mu} t_{\nu}^{*} Q_{v}=$ $t_{\mu \lambda_{s(\mu)}} t_{\nu \lambda_{s(\mu)}}^{*}$ whenever $\mu, \nu \in G \cap \Lambda^{n}$ with $s(\mu)=s(\nu)$, and (6.5) follows.

Combining (6.3), (6.2) and (6.5) proves the Proposition.

For the following proof, recall from the opening of [14, Section 3] that there is a strongly continuous action $\gamma: \mathbb{T}^{k} \rightarrow \operatorname{Aut}\left(C^{*}(\Lambda)\right)$ characterised by

$$
\gamma_{z}\left(s_{\lambda}\right)=z^{d(\lambda)} s_{\lambda}=z_{1}^{d(\lambda)_{1}} z_{2}^{d(\lambda)_{2}} \cdots z_{k}^{d(\lambda)_{k}} s_{\lambda}
$$

for all $\lambda \in \Lambda$. Averaging over this action yields a faithful conditional expectation $\Phi: C^{*}(\Lambda) \rightarrow \overline{\operatorname{span}}\left\{s_{\mu} s_{\nu}^{*}: d(\mu)=d(\nu)\right\}$ (see [14, Lemma 3.3]) such that $\Phi\left(s_{\mu} s_{\nu}^{*}\right)=$ $\delta_{d(\mu), d(\nu)} s_{\mu} s_{\nu}^{*}$ for all $\mu, \nu \in \Lambda$. 
Proof of Theorem 6.1. Follow the first paragraph of the proof of [14, Theorem 3.4] to see that $\pi$ is injective on $C^{*}(\Lambda)^{\gamma}=\overline{\operatorname{span}}\left\{s_{\mu} s_{\nu}^{*}: d(\mu)=d(\nu)\right\}$.

Proposition 6.3 implies that the formula

$$
\sum_{\mu, \nu \in F} a_{\mu, \nu} t_{\mu} t_{\nu}^{*} \mapsto \sum_{\mu, \nu \in F, d(\mu)=d(\nu)} a_{\mu, \nu} t_{\mu} t_{\nu}^{*}
$$

is well defined on finite linear combinations (if two linear combinations are equal, Proposition 6.3 implies that the norm of the difference of their images is zero), and normdecreasing, and hence extends by continuity to a linear map $\Psi: \pi_{t}\left(C^{*}(\Lambda)\right) \rightarrow \overline{\operatorname{span}}\left\{t_{\mu} t_{\nu}^{*}\right.$ : $d(\mu)=d(\nu)\}$ such that $\Psi\left(t_{\mu} t_{\nu}^{*}\right)=\delta_{d(\mu), d(\nu)} t_{\mu} t_{\nu}^{*}$.

To complete the proof, we argue as in the last two lines of the proof of [14, Theorem 3.4]: Let $\Phi$ be the faithful conditional expectation on $C^{*}(\Lambda)$ described above. By linearity and continuity, $\pi \circ \Phi=\Psi \circ \pi$. Suppose that $\pi(a)=0$. Then $\Psi\left(\pi\left(a^{*} a\right)\right)=0$ and hence $\pi\left(\Phi\left(a^{*} a\right)\right)=0$. Since $\pi$ is injective on $C^{*}(\Lambda)^{\gamma}$, it follows that $\Phi\left(a^{*} a\right)=0$. Since $\Phi$ is a faithful expectation, we then have $a^{*} a=0$ and hence $a=0$.

Let $\Lambda$ be a row-finite graph without sources. As in [15], we say that $\Lambda$ is cofinal if for every pair $v, w \in \Lambda^{0}$ there exists $n \in \mathbb{N}^{k}$ such that $v \Lambda s(\lambda) \neq \emptyset$ for all $\lambda \in w \Lambda^{n}$.

Remark 6.4. For row-finite graphs without sources, [15, Proposition A.2] implies that this notion of cofinality is equivalent to [15, Definition 3.3], and hence by [15, Theorem 5.1] to the usual one involving infinite paths.

Modulo the different formulation of cofinality, the following characterisation of simplicity appeared in [21], and was generalised to locally convex $k$-graphs in [22] and finitely aligned $k$-graphs in [15, 24].

Theorem 6.5. Let $\Lambda$ be a row-finite $k$-graph with no sources. Then $C^{*}(\Lambda)$ is simple if and only if $\Lambda$ is both aperiodic and cofinal.

In the proof we use the infinite-path representation. By [14, Proposition 2.3], for $x \in \Lambda^{\infty}, \lambda \in \Lambda r(x)$ and $n \in \mathbb{N}^{k}$, there are unique elements $\sigma^{n}(x)$ and $\lambda x$ of $\Lambda^{\infty}$ such that

$$
\sigma^{n}(x)(p, q)=x(n+p, n+q) \text { and }(\lambda x)(p, q)=(\lambda x(0, q))(p, q)
$$

for $p \leq q \in \mathbb{N}^{k}$. Let $\left\{\xi_{x}: x \in \Lambda^{\infty}\right\}$ be the usual orthonormal basis for $\ell^{2}\left(\Lambda^{\infty}\right)$. Then for each $\lambda \in \Lambda$ there is a partial isometry $S_{\lambda}$ on $\ell^{2}\left(\Lambda^{\infty}\right)$ such that $S_{\lambda} \xi_{x}=\xi_{\lambda x}$, and $\left\{S_{\lambda}: \lambda \in \Lambda\right\}$ is a Cuntz-Krieger $\Lambda$-family which gives a representation $\pi_{S}$ of $C^{*}(\Lambda)$ on $\ell^{2}\left(\Lambda^{\infty}\right)$.

The following lemma is a special case of the implication (ii) $\Longrightarrow$ (i) in $[15$, Theorem 5.1], but the proof simplifies significantly in our setting.

Lemma 6.6. Let $\Lambda$ be a row-finite $k$-graph with no sources which is not cofinal. Then there exist a vertex $v \in \Lambda^{0}$ and an infinite path $x \in \Lambda^{\infty}$ such that $v \Lambda x(n)=\emptyset$ for all $n \in \mathbb{N}^{k}$.

Proof. Since $\Lambda$ is not cofinal, there exist $v, w \in \Lambda^{0}$ such that, for each $n \in \mathbb{N}^{k}$, there exists $\lambda \in w \Lambda^{n}$ with $v \Lambda s(\lambda)=\emptyset$. Choose $n^{(i)} \rightarrow \infty$ in $\mathbb{N}^{k}$, and $\lambda_{i} \in w \Lambda^{n^{(i)}}$ such that $v \Lambda s\left(\lambda_{i}\right)=\emptyset$. Let $1_{k}=(1, \ldots, 1) \in \mathbb{N}^{k}$. Since $\Lambda$ is row-finite, there exists $\mu_{1} \in v \Lambda^{1_{k}}$ such that $S_{1}:=\left\{j \in \mathbb{N}: \lambda_{j}\left(0,1_{k}\right)=\mu_{1}\right\}$ is infinite. An induction argument now shows 
that there is a sequence $\left(\mu_{i}\right)_{i=1}^{\infty}$ in $v \Lambda$ such that for every $i \geq 2$, we have $\mu_{i} \in \mu_{i-1} \Lambda^{1_{k}}$, and $S_{i}:=\left\{j \in S_{i-1}: \lambda_{j}\left(0, i \cdot 1_{k}\right)\right\}$ is infinite. In particular, for any $i \in \mathbb{N}$ and $j \in S_{i}$ that $v \Lambda s\left(\lambda_{j}\right)=\emptyset$ forces $v \Lambda s\left(\mu_{i}\right)=\emptyset$. Since $d\left(\mu_{i}\right) \rightarrow(\infty, \ldots, \infty)$, [14, Remarks 2.2] imply that there is an infinite path $x$ such that $x(0, d(\mu-i))=\mu_{i}$ for all $i$. Now since $v \Lambda x\left(d\left(\mu_{i}\right)\right)=v \Lambda s\left(\mu_{i}\right)=\emptyset$ for all $i$, we have $v \Lambda x(n)=\emptyset$ for all $n$, so the infinite path $x$ has the required properties.

Proof of Theorem 6.5. First suppose that $\Lambda$ is aperiodic and cofinal, and let $I$ be a nonzero ideal in $C^{*}(\Lambda)$. To see that $I=C^{*}(\Lambda)$, we fix $\mu \in \Lambda$ and aim to show that $s_{\mu}$ belongs to $I$. Since $\Lambda$ is aperiodic, the Cuntz-Krieger uniqueness theorem (Theorem 6.1) implies that $I$ contains a vertex projection $s_{v}$. Applying cofinality with this $v$ and $w=s(\mu)$ gives $n \in \mathbb{N}^{k}$ such that for each $\lambda \in s(\mu) \Lambda^{n}$ there exists $\nu_{\lambda} \in v \Lambda s(\lambda)$. Then

$$
s_{\mu}=\sum_{\lambda \in s(\mu) \Lambda^{n}} s_{\mu \lambda} s_{s(\lambda)} s_{\lambda}^{*}=\sum_{\lambda \in s(\mu) \Lambda^{n}} s_{\mu \lambda}\left(s_{\nu_{\lambda}}^{*} s_{v} s_{\nu_{\lambda}}\right) s_{\lambda}^{*} \in I
$$

as required.

For the other direction, we first suppose that $\Lambda$ is not aperiodic, so that there exist $v \in \Lambda^{0}$ and distinct $m, n \in \mathbb{N}^{k}$ such that, for every $\lambda \in v \Lambda$ with $d(\lambda) \geq m \vee n$,

$$
\lambda(m, m+d(\lambda)-(m \vee n))=\lambda(n, n+d(\lambda)-(m \vee n)) .
$$

Then for every $x \in v \Lambda^{\infty}$ and $l \in \mathbb{N}^{k}$, we can apply (6.6) to $\lambda=x(0,(m \vee n)+l)$ and deduce that $x(m, m+l)=x(n, n+l)$, whence $\sigma^{m}(x)=\sigma^{n}(x)$.

We now fix $\lambda \in v \Lambda^{m \vee n}$, let $\mu=\lambda(0, m)$ and $\nu=\lambda(0, n)$, and aim to prove that $a:=s_{\lambda} s_{\lambda}^{*}-s_{\mu} s_{\nu}^{*} s_{\lambda} s_{\lambda}^{*}$ is nonzero and belongs to ker $\pi_{S}$; since we know that ker $\pi_{S}$ does not contain any vertex projections, this will prove that ker $\pi_{S}$ is a nontrivial ideal. To see that $\pi_{S}(a)=0$, fix $x \in \Lambda^{\infty}$ and compute

$$
\pi_{S}(a) \xi_{x}=\left(S_{\lambda} S_{\lambda}^{*}-S_{\mu} S_{\nu}^{*} S_{\lambda} S_{\lambda}^{*}\right) \xi_{x}
$$

If $x(0, d(\lambda)) \neq \lambda$, then (6.7) vanishes. If $x(0, d(\lambda))=\lambda$, then $x \in v \Lambda^{\infty}$, the argument in the previous paragraph gives $\sigma^{m}(x)=\sigma^{n}(x)$, and hence

$$
\nu \sigma^{n}(x)=x=\mu \sigma^{m}(x)=\mu \sigma^{n}(x),
$$

which implies $S_{\mu} S_{\nu}^{*} \xi_{x}=\xi_{\mu \sigma^{n}(x)}=\xi_{x}$ and $\pi_{S}(a) \xi_{x}=0$. Thus $\pi_{S}(a)=0$.

To see that $a \neq 0$, we choose $z \in \mathbb{T}^{k}$ such that $z^{m-n}=-1$. Then $\gamma_{z}\left(s_{\mu} s_{\nu}^{*} s_{\lambda} s_{\lambda}^{*}\right)=$ $-s_{\mu} s_{\nu}^{*} s_{\lambda} s_{\lambda}^{*}$, and hence

$$
\pi_{S}\left(a+\gamma_{z}(a)\right)=\pi_{S}\left(2 s_{\lambda} s_{\lambda}^{*}\right)=2 S_{\lambda} S_{\lambda}^{*} \neq 0,
$$

forcing $a \neq 0$. Thus $C^{*}(\Lambda)$ is not simple.

Now suppose that $\Lambda$ is not cofinal. By Lemma 6.6, there exist $v \in \Lambda^{0}$ and $x \in \Lambda^{\infty}$ such that $v \Lambda x(n)=\emptyset$ for all $n \in \mathbb{N}^{k}$. Let

$$
[x]_{\sigma}:=\left\{y \in \Lambda^{\infty}: \text { there exist } p, q \in \mathbb{N}^{k} \text { such that } \sigma^{p}(x)=\sigma^{q}(y)\right\} .
$$

We claim that

$$
y \in[x]_{\sigma} \Longrightarrow r(y) \neq v .
$$

To see this, fix $y \in[x]_{\sigma}$ and $p, q \in \mathbb{N}^{k}$ such that $\sigma^{p}(x)=\sigma^{q}(y)$. Then $x(p)=y(q)$ and hence $v \Lambda y(q)=\emptyset$ by choice of $x$. In particular, $y(0, q) \notin v \Lambda y(q)$, so $r(y) \neq v$, as claimed. 
We now consider the subspace $\mathcal{H}_{x}:=\overline{\operatorname{span}}\left\{\xi_{y}: y \in[x]_{\sigma}\right\}$ of $\ell^{2}\left(\Lambda^{\infty}\right)$. For $y \in[x]_{\sigma}$ and $s(\lambda)=r(y)$, we have $\lambda y \in[x]_{\sigma}$, and hence $\mathcal{H}_{x}$ is invariant for $S_{\lambda}$. On the other hand, $S_{\lambda}^{*} \xi_{y}$ vanishes unless $y(0, d(\lambda))=\lambda$, and then $S_{\lambda}^{*} \xi_{y}=\xi_{\sigma^{d(\lambda)}(y)}$, which also belongs to $\mathcal{H}_{x}$. Thus $\mathcal{H}_{x}$ is reducing for $\pi_{S}$, and $\phi_{x}:\left.a \mapsto \pi_{S}(a)\right|_{\mathcal{H}_{x}}$ is a homomorphism of $C^{*}(\Lambda)$ into $\mathcal{B}\left(\mathcal{H}_{x}\right)$. Since $\phi_{x}\left(s_{r(x)}\right) \xi_{x}=\xi_{x} \neq 0$, ker $\phi_{x}$ is not all of $C^{*}(\Lambda)$. Equation (6.8), on the other hand, implies that $\phi_{x}\left(s_{v}\right) \xi_{y}=0$ for all $y \in[x]_{\sigma}$, so $s_{v} \in \operatorname{ker} \phi_{x}$. Thus $C^{*}(\Lambda)$ is not simple.

\section{REFERENCES}

[1] G. Aranda Pino, J. Clark, A. Huef, and I. Raeburn, Kumjian-Pask algebras of higher-rank graphs, preprint 2011 (arXiv:1106.4361v1 [math.RA]).

[2] P. F. Baum, P. M. Hajac, R. Matthes, and W. Szymański, The K-theory of Heegaard-type quantum 3-spheres, $K$-Theory 35 (2005), 159-186.

[3] K. R. Davidson and D. Yang, Periodicity in rank 2 graph algebras, Canad. J. Math. 61 (2009), $1239-1261$.

[4] K. R. Davidson and D. Yang, Representations of higher rank graph algebras, New York J. Math. 15 (2009), 169-198.

[5] K. Deicke, J.H. Hong, and W. Szymański, Stable rank of graph algebras. Type I graph algebras and their limits, Indiana Univ. Math. J. 52 (2003), 963-979.

[6] D. Drinen, Viewing AF-algebras as graph algebras, Proc. Amer. Math. Soc. 128 (2000), 1991-2000.

[7] D.G. Evans, On the K-theory of higher-rank graph $C^{*}$-algebras, New York J. Math. 14 (2008), $1-31$.

[8] C. Farthing, P.S. Muhly, and T. Yeend, Higher-rank graph $C^{*}$-algebras: an inverse semigroup and groupoid approach, Semigroup Forum 71 (2005), 159-187.

[9] N.J. Fowler and A. Sims, Product systems over right-angled Artin semigroups, Trans. Amer. Math. Soc. 354 (2002), 1487-1509.

[10] E.R. Green, Graph products of groups, PhD Thesis, The University of Leeds, 1990. Online at http://etheses.whiterose.ac.uk/236/1/uk_bl_ethos_254954.pdf

[11] J.H. Hong and W. Szymański, Quantum spheres and projective spaces as graph algebras, Comm. Math. Phys. 232 (2002), 157-188.

[12] J.H. Hong and W. Szymański, The primitive ideal space of the $C^{*}$-algebras of infinite graphs, J. Math. Soc. Japan 56 (2004), 45-64.

[13] J.A. Jeong and G.H. Park, Graph $C^{*}$-algebras with real rank zero, J. Funct. Anal. 188 (2002), $216-226$.

[14] A. Kumjian and D. Pask, Higher rank graph $C^{*}$-algebras, New York J. Math. 6 (2000), 1-20.

[15] P. Lewin and A. Sims, Aperiodicity and cofinality for finitely aligned higher-rank graphs, Math. Proc. Cambridge Philos. Soc. 149 (2010), 333-350.

[16] D. Pask, J. Quigg, and I. Raeburn, Fundamental groupoids of k-graphs, New York J. Math. 10 (2004), 195-207.

[17] D. Pask, I. Raeburn, M. Rørdam, and A. Sims, Rank-two graphs whose $C^{*}$-algebras are direct limits of circle algebras, J. Funct. Anal. 239 (2006), 137-178.

[18] D. Pask, I. Raeburn, and N.A. Weaver, A family of 2-graphs arising from two-dimensional subshifts, Ergod. Th. \& Dynam. Sys. 29 (2009), 1613-1639.

[19] I. Raeburn, A. Sims, and T. Yeend, Higher-rank graphs and their $C^{*}$-algebras, Proc. Edinb. Math. Soc. (2) 46 (2003), 99-115.

[20] I. Raeburn, A. Sims, and T. Yeend, The $C^{*}$-algebras of finitely aligned higher-rank graphs, J. Funct. Anal. 213 (2004), 206-240.

[21] D.I. Robertson and A. Sims, Simplicity of $C^{*}$-algebras associated to higher-rank graphs, Bull. Lond. Math. Soc. 39 (2007), 337-344.

[22] D.I. Robertson and A. Sims, Simplicity of $C^{*}$-algebras associated to row-finite locally convex higherrank graphs, Israel J. Math. 172 (2009), 171-192. 
[23] H. Schubert, Categories, Translated from the German by Eva Gray, Springer-Verlag, New York, $1972, \mathrm{xi}+385$.

[24] J. Shotwell, Simplicity of finitely-aligned k-graph $C^{*}$-algebras, J. Operator Theory, to appear (arXiv:0810.4567v1 [math.OA]).

[25] J. Spielberg, Graph-based models for Kirchberg algebras, J. Operator Theory 57 (2007), 347-374.

[26] S.B.G. Webster, The path space of a higher-rank graph, Stud. Math. 204 (2011), 155-185.

Robert Hazlewood, School of Mathematics and Statistics, University of New South Wales, Sydney, NSW 2052, Australia

E-mail address: robbiehazlewood@gmail.com

Iain Raeburn, Department of Mathematics and Statistics, University of Otago, PO Box 56, Dunedin 9054, New Zealand

E-mail address: iraeburn@maths.otago.ac.nz

Aidan Sims and Samuel B.G. Webster, School of Mathematics and Applied Statistics, University of Wollongong, NSW 2522, Australia

E-mail address: asims@uow.edu.au, sbgwebster@gmail.com 